\title{
Frame und Framing: Frametheoretische Konsequenzen aus der Praxis und Analyse strategischen politischen Framings
}

\author{
Josef Klein
}

\begin{abstract}
Frame ist Struktur. Framing ist Handeln. Um beides kümmern sich unterschiedliche Wissenschaften, teilweise ohne einander zur Kenntnis zu nehmen. Framing, vor allem strategisches Framing, ist nicht Wissenschaft, sondern Praxis. Strategisches politisches Framing, insbesondere in Wahlkampagnen, ist kollektives Handeln politischer Akteure - gegebenenfalls unter Nutzung framewissenschaftlicher Erkenntnisse. Es zielt auf erwünschte Wissensbestände (= Überzeugungen, Meinungen und Einstellungen) bei Wählerinnen und Wählern. Insofern hängen Framing und Frames zusammen. Vor diesem Hintergrund sind mit dem Beitrag zwei Ziele verbunden: (1) den Zusammenhang zwischen Frames als ,Wissensrahmen und Framing als (kollektives) Handeln nicht nur an einzelnen Begriffen und Begriffsfeldern, sondern im Hinblick auf eine ganze politische Wahlkampagne frameanalytisch zu klären und (2) zu diesem Zweck einige frametheoretische Neuerungen, u. a. mit dem Einbezug von Emotionen, vorzulegen.
\end{abstract}

\section{Einleitung}

Die Kommunikationswissenschaft kennt seit zwei bis drei Jahrzehnten Framing als eigenen Forschungsgegenstand. Ihr Framing-Konzept ist, anders als man vielleicht erwarten würde, nicht präzis und konsistent aus der Frame-Theorie bzw. 
einer ihrer Ausprägungen heraus entwickelt worden. In einer jüngst erschienenen Darstellung der kommunikationswissenschaftlichen Framing-Forschung wird „Frame“ weit und etwas vage definiert:

Frames werden als ,Sinnhorizonte von Akteuren verstanden, die gewisse Informationen und Positionen hervorheben und andere ausblenden [...]. Frames (lassen sich) sowohl im kognitiven Apparat des Menschen ausmachen als auch in kommunizierten Inhalten. (Matthes 2014, 10)

Für „Framing“ als Handlung ist die Definition von Entman geradezu kanonisch geworden:

To frame is to select some aspects of a perceived reality and make them more salient in a communicating text, in such a way as to promote a particular problem definition, causal interpretation, moral evaluation and/or treatment recommendation for the item described. (Entman 1993, 52. Hervorhebung im Original)

Entman hatte zuvor die Bedeutung von Framing - nicht zuletzt mit kritischem Blick auf das journalistische Objektivitätsgebot - am Beispiel der Berichterstattung führender US-amerikanische Medien über zwei vergleichbare Abschüsse ziviler Verkehrsflugzeuge - eines koreanischen durch UdSSR-Militär und eines iranischen durch US-Militär - herausgearbeitet. Das erste Ereignis wurde durch Hervorhebung und sprachliche Konzeptualisierung bestimmter Sachverhalte mehr als verbrecherischer Akt, das zweite durch Betonung anderer Sachverhalte und durch unterschiedliche Konzeptualisierung eher als bedauerliches Versehen ,geframet‘ (Entman 1991).

Die kommunikationswissenschaftliche Framing-Forschung ist theoretisch und empirisch bislang fast ausschließlich an Framing als Praxis von Massenmedien und deren Wirkung interessiert („Medien-Framing“, vgl. Matthes 2014, 36 ff.). Strategisches Framing politischer Akteure, etwa in Wahlkampagnen oder ganz allgemein im Rahmen (partei-)politischer Framing-Konkurrenz, sind dort bisher stiefmütterlich behandelt worden. Das wird nicht zuletzt darin deutlich, dass die von Entman als Kernelemente des Framing hervorgehobenen vier Schritte zwar fundamentalen Elementen des für die Begründung politischen Handelns grundlegenden topischen Musters ${ }^{1}$ entsprechen: Situationsdaten (problem definition), Ursachen (causal interpretation), Situationsbewertung (moral evaluation) und als Schlussfolgerung Handeln/Handlungsempfehlung (treatment recommendation).

1 Der Begriff „topisches Muster“ verweist auf seine Herkunft aus Argumentationstheorie und Topik. 


\section{Frame und Framing}

Doch gerade hier zeigt sich der Unterschied zwischen Medienframing und politischem Framing. In Mediendarstellungen genügt es meist, Probleme und Ursachen zu benennen, sie zu bewerten und dann Handeln einzufordern. In strategischer Kommunikation politischer Akteure kommt bei der Begründung politischen Handelns Einiges hinzu:

- die Prinzipien offen zu legen, nach denen gewertet wird und gehandelt werden soll,

- die Ziele des Handeln zu bestimmen,

- die Konsequenzen von Nicht-Handeln oder anderem Handeln zu verdeutlichen.

Die politikwissenschaftliche und politolinguistische Argumentationsforschung hat an zahlreichen Texten, Reden und Diskursen zeit- und kulturübergreifend nachgewiesen: Auf persuasives Legitimieren politischen Handelns ausgerichtetes Framing umfasst durchweg eine Konstellation grundlegender Begründungselemente (Basistopoi), die folgendes ,topische Muster ${ }^{6}$ bilden: ${ }^{2}$

- Datentopos

- Ursachentopos

- Valuationstopos

- Prinzipientopos

- Finaltopos

- Konsequenztopos

Ausgerichtet ist die Konstellation der Topoi auf (vollzogenes, zugesagtes, gefordertes oder abgelehntes) politisches Handeln als Konklusion. (Frametheoretisch reformuliert bildet dieses Muster eine feste Konstellation von Attributen im Barsalou'schen Sinne, vgl. Barsalou 1992, Abschnitt 4.3 und 5.3.)

Auch mit einem weiteren Punkt, der bisherigen Nichtberücksichtigung von Emotionen, wird die auf Medien-Framing orientierte Kommunikationswissenschaft den Bedürfnissen politischer Framing-Praxis nicht gerecht (vgl. Kühne/ Schemer 2014, 195). Darin trifft sie sich mit der auf die kognitive Dimension

2 Vgl. Klein 1995, 2000, 2002, 2003, 2007; Kuhlmann 1999, 118-124; Kuck/Römer 2012; Römer/Wengeler 2013. Die Terminologie ist zwischen den Autoren nicht ganz einheitlich. Auch schwankt die Kategorisierung der kausalen Faktoren zwischen Spezifizierung als Ursachentopos (Kuck/Römer 2012, Römer/Wengeler 2013) und Zuordnung zu einem weit verstandenen Datentopos (Klein) oder zu einer „Situation“ (Kuhlmann 1999). 
(,Wissen') konzentrierten Frame-Theorie und ihren Ausprägungen. Bei den Akteuren politisch-strategischen Framings spielen emotionale Aspekte hingegen seit je eine praktische, allerdings nicht systematisch reflektierte Rolle. In der medienwissenschaftlichen Framing-Forschung werden Emotionen in jüngster Zeit zwar beachtet. Es handelt sich allerdings nur um wenige Emotionen, insbesondere Ärger und Trauer. Die Ergebnisse gehen noch kaum über die Bestätigung alltagsweltlichen Erfahrungswissens durch empirisch-sozialwissenschaftliche Methoden hinaus. So weisen Kühne und Schemer (2014) nach, dass Berichte über negativ bewertete Sachverhalte, die in der Verantwortung von Akteuren liegen, Ärger (über die Akteure) auslösen, während auf Berichte über schlimme Ereignisse, für die niemand verantwortlich ist, z. B. Naturkatastrophen, nicht mit Ärger, sondern mit Traurigkeit reagiert wird.

\section{Framing in der politischen Beratung}

Strategisches Framing in der Politik

- zielt auf Wissen in einem weiten Sinne, insofern es Einstellungen einschließt,

- strebt gesellschaftliche Durchsetzung an,

- steht im Konkurrenz mit gegnerischen Frames,

- ist an framesemantische Konzepte anschließbar,

- geht über die Lexem-Ebene hinaus,

- wird auf unterschiedlichem Niveau praktiziert.

Den letzten Punkt greife ich zunächst auf: das wissenschaftliche, intellektuelle und professionelle Niveau, auf dem strategisches Framing insbesondere im Bereich der Politikberatung betrieben wird - zunächst in den USA, dann in Deutschland.

Der weltweit bekannteste politische Framing-Berater ist der Linguist George Lakoff. Über sein wissenschaftliches Niveau als Theoretiker im Bereich der Kognitiven Linguistik, vor allem als Vater des modernen Metaphernverständnisses, braucht hier nichts weiter ausgeführt zu werden. Seit Ende der 1990er Jahre ist er mehr und mehr mit dem Anspruch als Politikberater für das ,progressive', dem linken Flügel der Demokraten zugerechnete ,Lager“ in den USA aufgetreten zeitweilig im Rahmen des eigens zu diesem Zweck gegründetes Rockridge Institute (1997-2008). In dem zeitgleich in Englisch und Deutsch erschienenen Band 
„Auf leisen Sohlen ins Gehirn. Politische Sprache und ihre heimliche Macht“ (Heidelberg 2008) lässt Lakoff sich von der deutschen Mitarbeiterin Elisabeth Wehling interviewen und macht sein Konzept von Frame und Framing deutlich:

Es gibt zwei Arten von Frames: ,Surface Frames', durch die wir die Bedeutung einzelner Worte und Sätze erfassen, also Frames auf der sprachlichen Ebene, und ,Deep Seated Frames'. Deep Seated Frames sind in unserem Gehirn tiefverankerte Frames, die unser generelles Verständnis von der Welt strukturieren, unsere Annahmen von der Welt zum Beispiel auf Grund unserer moralischen und politischen Prinzipien, und die für uns schlicht ,wahr ${ }^{\text {s }}$ sind - die also unseren eigenen Common Sense ausmachen. (Lakoff/Wehling 2008, 73)

Für die USA nimmt er - empirisch wenig abgesichert - zwei konkurrierende Deep Seated Frames an. Beide modellieren Politik als Familiengeschehen. Danach haben die Konservativen den ,Strenge-Vater-Frame“ und die Liberalen den ,Fürsorgliche-Eltern-Frame' verinnerlicht.

Lakoff arbeitet nicht exklusiv für bestimmte Politiker, sondern wendet sich mit seinen Publikationen an die ,progressive' Öffentlichkeit. Er stellt einen - wenig selbstkritischen - aufklärerischen Anspruch, und ähnelt darin der europäischen sog. ,kritischen Diskursanalyse'. Mit der hat er auch gemeinsam, dass er sich fast ausschließlich mit der Destruktion von Sprachstrategien des politischen Gegners - bei ihm die Republikaner - beschäftigt. Dazu ein Beispiel, das in Lakoffs Publikationen immer wieder auftaucht. Es betrifft die Steuerpolitik der Republikaner:

Denken Sie an die Bezeichnung ,Tax Relief', also ,Steuererleichterung ${ }^{\prime}$ - das ist ein sprachlicher Frame, der einem Denken im Sinne konservativer ,Strenge-Vater'Moral entspringt. Von dem Tag an, an dem George W. Bush das Präsidentenamt übernahm, tauchte der Begriff ,Steuererleichterung' in den Reden konservativer Politiker, den konservativen Medien und den Pressemitteilungen des Weißen Hauses auf. [...] Das Wort ,Erleichterungen` erweckt einen Frame, in dem es eine unschuldige Person gibt, die eine Last tragen muss und dadurch in Bedrängnis gerät. Die Last ist ihr von außen auferlegt worden. Man kann die Person von der Last befreien. Indem man das tut, erlöst man die Person. [...]

Sooft wir das Wort ,Steuererleichterung ' hören, wird dieser Frame aktiviert und mit der Zeit Teil unseres politischen Common Sense. Steuern sind eine Last, und die konservative Partei ist diejenige, die uns heldenhaft von dieser Last befreien will. Im Umkehrschluss werden die Progressiven zu denen, die den Bürger durch Steuern belasten, ihm also schaden. (ebd., 79 f.) 
So intensiv sich Lakoff mit Framing-Tricks der politischen Gegner befasst - genau genommen fast ausschließlich mit Metaphorik auf Lexikonebene - so sparsam und wenig konkret bleibt er mit Empfehlungen für ,progressives` Framing. Das gilt selbst für sein Lieblingsthema Steuerpolitik:

Nun, die Progressiven sollten in solchen Frames über Steuern sprechen, denen das Denken im Sinne des ,Commonwealth-Prinzips‘ zu Grunde liegt. Sie müssen die Idee enthalten, dass wir das gemeinsame Vermögen für unser gemeinsames Wohl nutzen, dass unser aller individuelle Träume und Ziele erheblich von diesem gemeinsamen Vermögen abhängen. [...] Man kann Frames schaffen, in denen Steuern nicht als Abgabe unseres privaten Vermögens an den Staat begriffen werden, sondern als Rückerstattung dessen, was die Gesellschaft uns im Vorwege zur Verfügung gestellt hat. (ebd., 81)

Es bleibt bei solch allgemeinen politisch-moralischen Hinweisen. Politikern ist das normalerweise zu wenig. Sie brauchen Formulierungsvorschläge.

Ganz anders Frank Luntz, der prominenteste Framing-Experte auf rechts-republikanischer Seite. Er ist spezialisiert auf Kreieren, Testen und Erklären wirksamen Framings für sein eigenes politisches Lager. Luntz kümmert sich wenig um die Sprache der Gegenseite. Beim Framing beschränkt er sich nicht auf die Wortebene, sondern bezieht einprägsame Sätze und deren Kontextualisierung ein. Typisch ist eine Passage des Kapitels „TAX RELIEF \& SIMPLIFICATION“ aus einem (lange Zeit nicht öffentlich zugänglichem) Handbuch für republikanische Politiker („The New American Lexicon“) (Luntz 2006, 52-65):

- You may be tempted to talk about tax policy in terms of reform. Don't. When Americans hear the word "reform", they fear that they will end up paying more. Far better for you to talk about "simplification" - which everyone supports and sees a benefit.

- You may be tempted to talk about making the tax cuts from 2001 and $2003^{3}$ "permanent". Don't. It is a far more effective to talk about "the largest tax increase in American history if these tax cuts are revoked". Remember, the American public dislikes a tax hike more than they like a tax cut.

- You may be tempted to talk about Americans are overtaxed overall. Do, but also emphasize that Washington spends too much as well. The more you link high taxes to high spending, the greater the support for tax relief.

If there is one debate, where framing the issue is as important as the policy itself, this is it.

3 Nach den Terroranschlägen vom 11. September 2001 hatte die Regierung Bush die Steuern zweimal gesenkt. 
Es folgen: (1) ein Katalog gängiger Stereotypen zur Politik und zum amerikanischen Selbstverständnis als Kontexte für darauf zugeschnittene Sätze zur Propagierung republikanischer Steuerpolitik, (2) Verknüpfung des republikanischen Steuerkonzepts mit den Prinzipien fairness, simplicity und reliability, ergänzt durch exemplarische Kontexte und Formulierungen, (3) Textausschnitte aus erfolgreichen Politikerreden zum Thema (Words that work).

Formulierungen, geeignet zur wörtlichen Übernahme, abgestimmt auf gängige Denk- und Situationskontexte - das können Politiker brauchen. Empirische Basis sind Texte, die sich als erfolgreich bewährt haben, sowie Tests in Focus-Gruppen. Luntz ist kein Frametheoretiker. Seine Praxis - beileibe nicht seine Ideologie scheint mir am ehesten an Dietrich Busses Frame-Konzept anschließbar: Frame als kulturell generierte kognitive Größe, die sich in sprachlichen Einheiten und Kombinationen auf mehreren linguistischen Strukturebenen vom Wort bis zum Text manifestiert (Busse 2012, 372 u. öfter).

In den deutschen Parteizentralen ist das Formulieren politischer Texte, auch von Kampagnentexten, nach Vorgaben durch die politische Führung verteilt auf die Abteilungen ,Politische Planung,, ,Presse- und Öffentlichkeitsarbeit' und die Redenschreiber/innen für die Chefs/Chefinnen. Es handelt sich fast ausschließlich um ehemalige Journalisten/Journalistinnen, selten mit sprachwissenschaftlicher Ausbildung. Meiner Erfahrung nach wird dort die Bezeichnung „Framing“ ungenau und mit unterschiedlicher Extension verwendet, mal eng als modischer Terminus für die Auswahl einzelner parteigünstiger Begriffe (eine Unterart des seit langem bekannten ,Begriffe-Besetzens', vgl. Klein 1991, 51 ff., auch 2014, $103 \mathrm{ff}$.), mal etwas weiter als Knüpfen von Begriffsnetzen und damit näher bei linguistischen Frame-Konzepten, gelegentlich auch als Bezeichnung für die sprachliche Gesamtgestaltung einer Wahlkampagne.

Damit liegen die politischen Kommunikationspraktiker jeweils partiell nicht falsch, insofern sich Mikro-, Meso- und Makroebene des Framings unterscheiden lassen - so in der überaus erfolgreichen Wahlkampagne der CDU zur Bundestagswahl am 22.09.2013, in der eine neue Stufe der Reflexion und Systematisierung strategischen Framings in Deutschland erreicht wurde. Deren framewissenschaftliche Modellierung wird Thema der folgenden Abschnitte sein. 


\section{Frametheoretische Vorüberlegungen und Weiterungen}

Die größte Herausforderung für strategisches politisches Framing stellen Wahlkämpfe dar. Wie die Praxis - auch als Herausforderung an die Wissenschaft aussieht, soll am Beispiel der Wahlkampagne der CDU im Bundestagswahlkampf 2013 erläutert werden. Ich habe diese Kampagne ausgewählt, zum einen weil sie mit 41,5 \% der Stimmen für die Union, darunter laut Infratest Dimap 1,25 Millionen bisherige Nichtwähler, besonders erfolgreich war, zum anderen weil ich als wissenschaftlicher Berater der Arbeitsgruppe ,Framing', die die CDU zur Vorbereitung der Kampagne gegründet hatte, einen guten Einblick in die Praxis gewonnen habe. ${ }^{4}$

Politisches Framing startet nicht von der Nulllinie. Ausgangspunkt ist weniger das Bild, das die Partei sich von der Politik macht, sondern das Bild, das sich soweit für die Kampagnenakteure ermittelbar bzw. plausibel rekonstruierbar die für die Partei relevanten Wahlberechtigten von der Politik, von den Parteien und deren Spitzenkandidaten machen. In frametheoretischer Terminologie handelt es sich um einen aus Kenntnissen und Meinungen bestehenden Wissensrahmen, von dem man annimmt, dass er im parteirelevanten Wählerspektrum ${ }^{5}$ ziemlich kollektiv gilt. Diesen soll die Kampagne zu einem im Sinne der Parteiziele optimierten Wissensrahmen umbauen. Frametheoretisch gewendet bedeutet es, dass Framing-Forschung hier der frameanalytischen Modellierung beider Wissensrahmen bedarf.

Während Framing auf der Mikroebene (gezielter Umgang mit Einzelbegriffen) und auf der Mesoebene (strategische Generierung und Behandlung von Begriffsnetzen) naheliegende Gegenstände für die framesemantische Analyse sind, gilt das für die Makroebene der Gesamtkampagne nicht ohne weiteres. Denn dazu gehören Wissensbestände, die nicht fest an bestimmte sprachliche Strukturen gebunden sind und daher eher als Gegenstände für Inhaltsanalysen in Frage zu kommen scheinen. Auch visuelles Framing, z. B. Plakatgestaltung, gehört dazu.

4 Vor diesem Hintergrund sind CSU-spezifische, auf Bayern abgestellte Züge innerhalb der Kampagne in diesem Beitrag nicht einbezogen.

5 Nicht alle Wähler gehören zum für eine Partei relevanten Wählerspektrum. Bei einer, Volkspartei‘ ist dieses zwar ziemlich breit, doch gehören diejenigen nicht dazu, die grundsätzlich konträren Positionen und Prinzipien anhängen. Um sie wird nicht geworben, auch nicht per Framing. 


\section{Frame und Framing}

Dennoch nutze ich das framesemantische Instrumentarium, wie es im Kompendium Frame-Semantik von Dietrich Busse entfaltet und weiter entwickelt wird (Busse 2012), auch für die Modellierung auf der Makroebene. Dafür sind drei Gründe ausschlaggebend: Erstens sind die meisten Züge strategischen Framings auch auf der Makroebene sprachlicher Natur - von der Zuspitzung von Inhalten in Slogans über deren Entfaltung in Reden bis zur Wahl bestimmter Verständlichkeitsniveaus; vor allem aber pflegen sie von den Akteuren - ähnlich wie in Diskursen $^{6}$ - immer wieder in Begriffen zusammengefasst und ,auf den Punkt gebracht zu werden. ${ }^{7}$ Zweitens erscheinen mir Frames, verstanden als Darstellungsformat, für die analytische und gleichzeitig übersichtliche Repräsentation von Wissensbeständen generell bestens geeignet, auch wenn das Wissen nicht fest an bestimmte sprachliche Strukturen gebunden ist. Und - damit zusammenhängend - ist drittens der Kernbestand des frameanalytischen Instrumentariums - abgesehen von Fillmore und Konerding - nicht von Linguisten, sondern vom KI-Forscher Minsky und vom Kognitionspsychologen Barsalou entwickelt worden.

Der äußeren Form nach handelt es sich bei den im Folgenden präsentierten Frames um instantiierte, an der CDU-Kampagne exemplifizierte Konzept-Frames, insofern die Modellierung vor allem in Form nominaler Begriffe erfolgt. Das geschieht im Bewusstsein, dass die Relationen zwischen ihnen prädikativen Charakter haben (dazu Busse 2012, 550-553 und 687-703).

Vor diesem Hintergrund erfordert die Eigenart des Gegenstandes einige Erweiterungen des bisherigen frametheoretischen Rahmens. Sie betreffen vor allem

(1) die Art des Wissens,

(2) das Kriterium zur Auswahl der in Frames repräsentierten Wissensbestände,

(3) die Relationen zwischen den Ebenen der ,Attribute“ und der ,Werte“ (nach Barsalous Terminologie, die hier der sehr ähnlichen Unterscheidung von ,slots' und ,fillers“ des frühen Minsky vorgezogen wird, vgl. Barsalou 1993, Minsky 1974),

(4) den frametheoretischen Umgang mit Emotionen.

$6 \mathrm{Zu}$ Gemeinsamkeiten und Unterschieden zwischen Kampagnen und Diskursen vgl. Klein (2014, $295 \mathrm{ff}$.)

7 Das ist auch die - gelegentlich implizite - Prämisse der frame-analytischen Tradition vor allem im deutschen Sprachraum, insbesondere Fraas (1996), Klein/Meißner (1999), Holly (2001), Klein (2002), Busse (2008), Ziem (2008). 


\subsection{Wissenstypus und Methode}

Frametheoretiker erläutern ihren Frame-Begriff durchweg an reduktionistischen Modellen. Dabei werden Frames fast ausschließlich an überindividuell und übersituativ geltenden Wissenseinheiten, repräsentiert durch Einzelbegriffe (Konzepte) oder satzförmige Prädikationen, exemplifiziert. So bleiben Fragen am Rand, die sich bei der Modellierung weniger simpel strukturierten Wissens stellen, etwa eines Wissens, das weit über das Fassungsvermögen des Kurzzeitgedächtnisses hinausgeht, oder eines Wissens, das sozio-kommunikativ als einheitlicher Wissenskomplex funktioniert, obwohl es aufgrund der kommunikativen Dynamik innerhalb von Gruppen keineswegs in identischen und präzisen Begriffen oder Satzmustern versprachlicht ist, sondern sich in vielerlei mündlichen oder schriftlichen Sprachakten und Texten, u. U. auch in visuellen Medien als intern und extern unscharf abgegrenztes holistisches Phänomen herausbildet.

Um solches Wissen handelt es sich in Fall einer Wahlkampagne. Zwar lässt sich das Lexem Wahlkampagne - frametheoretisch als ,Kategorie` im Sinne Barsalous verstanden - leicht als Konzept-Frame mit den Attribut-Wert-Konstellationen ,AKTEUR: Partei', ,WAHLWERBUNG: Plakate, Wahlreden etc.', ,ADRESSATEN: Wähler(innen)' repräsentieren. Allerdings ist keineswegs sicher, wie viel Allgemeinverbindlichkeit dieser Frame beanspruchen kann - schließlich kontextualisieren viele politisch Interessierte den Begriff Wahlkampagne auch mit Attributen wie SPITZENKANDIDAT(IN), RESSOURCEN oder FINANZIERUNG. Die Situation wird noch uneinheitlicher, wenn man eine Frame-Modellierung nicht auf der Abstraktionsebene von Konzepten, sondern auf der Ebene von Exemplaren eines Konzepts vornimmt. ${ }^{8}$ So verhält es sich mit der ,CDU-Bundestagswahlkampagne 2013'. Wenn man dabei das Interesse nicht auf das Wissen einer mehr oder weniger politisch interessierten Öffentlichkeit richtet, sondern auf das kampagnenbezogene Wissen, das eine Gruppe von Experten, hier die der politischen und kommunikationsstrategischen Akteure, ihren Wahlkampf-Aktivitäten zugrunde legt, hat man es mit einem Gegenstand zu tun, der sich im intertextuellen Geflecht aus demoskopischen Umfragen, Beschlüssen und Protokollen oberster Parteigremien, Texten der Planungs- und Kommunikationsabteilungen, Skizzen und Powerpoint-Folien von Beratern und Agenturen konstituiert. Es

8 Vgl. Busses (2012, 613 ff.) Unterscheidung von Muster- und Exemplar-Frames. 
handelt sich um einen Komplex kognitiv-kommunikativer Natur, für dessen frameanalytische Repräsentation zwar nicht der Anspruch einer genauen Abbildung bestimmter psychischer Prozesse oder kommunikativer Ereignisse erhoben werden kann. Doch will man das, was sich da in der strategischen Planungskommunikation durchsetzt und schließlich in den Köpfen der Akteure als gemeinsames Konstrukt dominiert, übersichtlich zusammenfassen und gleichzeitig analytisch vertiefend präsentieren, so bietet sich eine frameanalytische Modellierung an. ${ }^{9}$ (Für diese ist hier als Format die Tabellenform mit Zeilen, Spalten und Symbolen zur Indizierung von Relationen zwischen Frameelementen gewählt.)

Lässt man das Wissen zu Organisations- und Finanzierungsfragen, für die es eigene Experten gibt, beiseite, so bezieht sich das hier interessierende Wissen vor allem auf die Situation vor Beginn des Wahlkampfs (Ausgangsrahmen) und auf eine - angestrebte - Situation am Wahltag (Zielrahmen). Ausgangspunkt ist weniger das Bild, das sich die Führungselite der Partei vom Spektrum der politischen Sachprobleme macht, als das Politik- und Kandidatenbild in den Köpfen der für die Partei relevanten Wahlberechtigten. Es gilt also, im Wesentlichen Wissen über Wissen frameanalytisch zu modellieren, genauer: Kenntnisse und Annahmen der Kampagnenakteure über Kenntnisse, Annahmen und Einstellungen der für die Partei relevanten Wählerschaft.

Strategisches Framing besteht darin, den Ausgangsrahmen durch die Kampagne zu transformieren in einen Zielrahmen, der möglichst viele Wähler motiviert, die betreffende Partei - hier die CDU - zu wählen. Daher gehört es zu den zentralen Aufgaben der Framing-Akteure, die beiden Rahmen - sozusagen Ist und Soll - im Hinblick auf Gegebenheiten und Chancen möglichst realistisch zu entwerfen.

Vor dem frameanalytischen Zugriff stellt sich die methodische Frage, wie man sich der skizzierten Art von Wissen als Gegenstand einer wissenschaftlichen Untersuchung mit empirischem Anspruch nähern kann. Ich will die Frage nicht mit dem Anspruch auf Allgemeingültigkeit beantworten - da müsste man über die Leistungsfähigkeit von Interview, Korpus-Analyse, Inhaltsanalyse u. Ä. reden,

9 Um Modellierung (und nicht um schlichte Eins-zu-eins-Repräsentation) handelt es sich schon allein deshalb, weil in den scharfen begrifflichen Abgrenzungen der Elemente von Frame-Darstellungen Unschärfen, Überlappungen und Geltungsdifferenzen, die es in sozio-kommunikativen Konstrukten trotz aller Deutlichkeit von Dominanzen stets gibt, nicht repräsentiert werden können. 
aber auch über deren Grenzen für ,von außen' kommende Wissenschaftler/innen angesichts der Dynamik der Wissensgenerierung der Akteure und der Lückenhaftigkeit, Unübersichtlichkeit und beschränkten Zugänglichkeit relevanter Daten. Als partiell beteiligter Akteur setze ich demgegenüber auf eine Mischung aus teilnehmender Beobachtung, Zeugenbericht und Textanalyse - stets im Bewusstsein der Unhintergehbarkeit des hermeneutischen Charakters jeden Zugriffs auf sozio-kommunikative Wissensgenerierung und deren Ergebnisse.

\subsection{Relevant statt default}

Das Kriterium, unter dem Wissen in Frames vor allem in der Minsky-BarsalouTraditionslinie repräsentiert wird, ist Normalfallwissen (defaults). In kampagnentypischen Wissensrahmen tritt Normalfall-Wissen zurück hinter (unter wahlstrategischem Aspekt) für relevant gehaltenes Wissen. Die Relevant-Setzung erfolgt per Komplexitätsreduktionen auf zwei Ebenen: Da ist zunächst das Wissen der Akteure über die wahlrelevanten Aspekte von policy, polity und politics - wie jedes Wissen über politische, gesellschaftliche, kulturelle Phänomene eine soziokommunikativ erzeugte Konstruktion (Berger/Luckmann 1966; Searle 1997). Da sind ferner - als Teil dieses Konstrukts - mehr oder weniger empirisch fundierte Hypothesen über die politischen Kenntnisse und Einstellungen der für die jeweilige Partei relevanten Wählerschaft. Die Reduktion betrifft dabei zum Einen entsprechend der kampagnentypischen Konzentration auf wenige Sachthemen und auf den Spitzenkandidaten / die Spitzenkandidatin - sachpolitisches und personenbezogenes Wissen. Zum anderen wird die - gegenüber dem gesamten Elektorat schon reduzierte - parteirelevante Wählerschaft gleichsam kondensiert zu dem Wähler / der Wählerin, einer Art Kollektivperson auf der Basis angenommener Durchschnittswerte in den für Wahlverhalten relevanten Dimensionen. ${ }^{10} \mathrm{Re}-$ duziert wird - in teils systematischen, teils unsystematischen Kommunikationsprozessen zwischen den Akteuren (s. o.) - unter dem Aspekt der Relevanz für den Wahlerfolg. Default-Wissen spielt dabei lediglich eine dienende Rolle.

\footnotetext{
${ }^{10}$ Die Differenzierung der Wählerschaft in unterschiedliche Zielgruppen findet in Wahlkampagnen zwar regelmäßig statt, ist aber zumindest in Kampagnen von Parteien mit Volkspartei-Anspruch nachrangig.
} 


\subsection{Relationen}

Der relationale Charakter der in Frames erfassten Wissensbestände wird in den älteren Modellen der frametheoretischen Tradition nicht hinreichend erfasst. Erst Barsalou setzt sich mit der Betonung von ,strukturellen Invarianten' und ,Constraints' davon ab. In den unten präsentierten Frames wird dies aufgegriffen, indem Konstellationen zusammengehöriger Frameelemente als strukturelle Invarianten sowie Ranking-, Kausal- und Folgerungs-Relationen insbesondere zwischen Werten als Constraints identifiziert werden. ${ }^{11}$

Es besteht eine Neigung, das Verhältnis von Kategorie und Attribut nach dem Modell der Ganzes-Teil-Beziehung bei Konkreta zu konzeptualisieren. Das ist bei Abstrakta wie etwa dem Begriff „Eurokrise“, der in der Modellierung der CDUKampagne eine Rolle spielt, unangemessen. Bei den Attributen LAGE und VERANTWORTLICHKEIT zur Kategorie „Eurokrise“ handelt es sich nicht um Teile, sondern - wesentlich abstrakter - um Aspekte des Konzepts Eurokrise. Noch deutlicher wird dieses Defizit, sobald in Frames Klasse-Element-Beziehungen auftauchen, z. B. die Kategorie ,Politikfeld“ als Klasse mit den Attributen EUROKRISE, SOZIALE GERECHTIGKEIT und WIRTSCHAFT/ARBEITSMARKT als Elementen. Ähnliches gilt für das Verhältnis der Klasse der Emotionen zur einzelnen Emotion, etwa im Rahmen einer Attribut-Wert-Zuordnung des Typs EMOTION-Ärger. Die Systematisierung von Wissensbeständen nach einem Zweistufen-Modell wie bei Barsalou und Minsky auf der Basis von Rekursivität bedarf eines übergreifenden Begriffs wie ,Zuweisungsrelation', der nicht nur unterschiedliche Begriffspaare wie Ganzes - Teil, Konzept - Aspekt, Klasse - Element umfasst, sondern auch den kognitiv-hermeneutischen Akt der Zuweisung des einen Konzepts zum anderen verdeutlicht - Busse nennt das „epistemische Prädikation“ (ebenda, 687 ff.).

11 Allerdings weist Busse darauf hin, dass auch Barsalou den relationalen Charakter von Frames nicht konsequent beachtet, insbesondere bezüglich der „Zuordnungsleistung“ bzw. dem prädikativen Charakter von Attributen im Verhältnis zu ihrer jeweiligen Kategorie (Busse 2012, 369). 


\subsection{Emotionen}

Emotionen sind in der kognitiv orientierten Frame-Forschung bislang weitgehend unbeachtet geblieben. ${ }^{12}$ Solange die Frametheorie ihren Gegenstand nicht über,Wissen ' hinaus ausdehnt, wird Emotion als mentales bzw. physiologisches Geschehen - ebenso wie Handeln als performatives Geschehen - außerhalb bleiben. Aber es gibt keinen Grund, Wissen über Emotionen frametheoretisch und frameanalytisch zu ignorieren. Genau darum geht es aber beim strategischen politischen Framing: Wissen über Emotionen zu nutzen. Denn es gilt als ausgemacht, dass in der Einstellung von Wählern zu Politik, Parteien und deren Spitzenpersonal Emotionen eine wichtige Rolle spielen. In den für die CDU-Kampagne prägenden wählerbezogenen Wissensrahmen wurde der Aspekt ,Emotion systematisch berücksichtigt. Es ist - wahrscheinlich auch für den Erfolg der Kampagne - aufschlussreich, welche Emotionen die Akteure vor Beginn des Wahlkampfs als relevant identifizieren und welche für die Modellierung des Zielrahmens eine Rolle spielten. Um dies zu verdeutlichen, wird in Übersicht 1 eine Taxonomie der für politische Kommunikation wichtigsten Emotionen präsentiert. Zwar gibt es in der Psychologie weder eine Einigung darüber, welche dieser Emotionen als grundlegend $\mathrm{zu}$ gelten haben (sog. ,Basis-Emotionen') und welche nicht, noch darüber, welche Kriterien für eine allgemeingültige Einteilung von Emotionsklassen als entscheidend zu gelten haben (dazu Schwarz-Friesel 2007, 66 ff.). Für den Zweck politisch-strategischen Framings ist das allerdings insofern unerheblich, als die drei Kriterien, nach denen Emotionen in dieser Taxomie eingeteilt werden (Wertigkeit, Bezug, Stärkegrad), in der Emotionsforschung als solche kaum umstritten sind - unabhängig davon, für wie grundlegend man sie unter emotionstheoretischer Perspektive hält.

Bei der Kategorisierung und Klassifikation von Emotionen ist man auf den Emotionswortschatz natürlicher Sprachen, in unserem Falle des Deutschen angewiesen. Da manifestiert sich die Stärke-Ausprägung von Emotionen nicht immer in unterschiedlichen Begriffen. In manchen Fällen lässt sich unterschiedliche Stärke einer Emotion kaum anders als durch attributive oder ähnliche Spezifizie-

$12 \mathrm{Zu}$ den wenigen Hinweisen auf Emotionen gehört Barsalous Bemerkung: „By selecting the aspects of introspecting experience that are associated with anxiety, tranquillity, anger, and so forth, schematic representations of affective states develop" (Barsalou 1993, 54. Hervorhebung dort). 
rung des jeweiligen Emotionsbegriffs ausdrücken. Selbstbewusstsein, Minderwertigkeitsgefühl, Mitgefühl, Verachtung und Neid sind solche Begriffe (starkes Selbstbewusstsein, tiefste Verachtung, ein wenig Neid u. Ä.). In Übersicht 1 sind diese Emotionsbegriffe unter der Rubrik „moderat“ eingeordnet. Pfeile („<“ und „,“) signalisieren, dass sie stark oder schwach ausgeprägt sein können

\begin{tabular}{|c|c|c|c|c|c|c|}
\hline \multirow[t]{2}{*}{ Bezug } & \multicolumn{3}{|c|}{,positive' Emotionen } & \multicolumn{3}{|c|}{,negative' Emotionen } \\
\hline & stark & moderat & schwach & stark & moderat & schwach \\
\hline \multirow{2}{*}{$\begin{array}{l}\text { SITUA- } \\
\text { TION / } \\
\text { OBJEKT }\end{array}$} & $\begin{array}{l}\text { Eu- } \\
\text { phorie }\end{array}$ & $\begin{array}{l}\text { Hoffnung, } \\
\text { Zuversicht }\end{array}$ & $\begin{array}{l}\text { Erleichte- } \\
\text { rung }\end{array}$ & $\begin{array}{l}\text { Angst, } \\
\text { Furcht }\end{array}$ & Sorge & $\begin{array}{l}\text { Unbehagen, } \\
\text { Skepsis }\end{array}$ \\
\hline & $\begin{array}{l}\text { Be- } \\
\text { geiste- } \\
\text { rung }\end{array}$ & Freude & $\begin{array}{l}\text { Zufrieden- } \\
\text { heit }\end{array}$ & $\begin{array}{l}\text { Ver- } \\
\text { zweif- } \\
\text { lung }\end{array}$ & $\begin{array}{l}\text { Traurig- } \\
\text { keit }\end{array}$ & $\begin{array}{l}\text { Deprimiert- } \\
\text { heit }\end{array}$ \\
\hline \multirow{2}{*}{ SELBST } & Stolz & $\begin{array}{l}\text { Selbstver- } \\
\text { trauen }\end{array}$ & $\begin{array}{l}\text { Selbstzu- } \\
\text { friedenheit }\end{array}$ & Scham & $\begin{array}{l}\text { Schuld- } \\
\text { gefühl }\end{array}$ & $\begin{array}{l}\text { Gewissens- } \\
\text { bisse }\end{array}$ \\
\hline & $<$ & $\begin{array}{l}\text { Selbstbe- } \\
\text { wusstsein }\end{array}$ & $>$ & $<$ & $\begin{array}{l}\text { Minder- } \\
\text { wertig- } \\
\text { keits- } \\
\text { gefühl }\end{array}$ & $>$ \\
\hline \multirow{3}{*}{$\begin{array}{l}\text { AN- } \\
\text { DERE }\end{array}$} & $\begin{array}{l}\text { En- } \\
\text { thusi- } \\
\text { asmus }\end{array}$ & Vertrauen & $\begin{array}{l}\text { Achtung, } \\
\text { Wohlwol- } \\
\text { len }\end{array}$ & $\begin{array}{l}\text { Wut, } \\
\text { Zorn }\end{array}$ & $\begin{array}{l}\text { Ärger } \\
\text { Miss- } \\
\text { trauen }\end{array}$ & Unmut \\
\hline & Liebe & Sympathie & Zuneigung & Hass & $\begin{array}{l}\text { Antipa- } \\
\text { thie }\end{array}$ & Abneigung \\
\hline & $<$ & Mitgefühl & $>$ & $<$ & $\begin{array}{l}\text { Verach- } \\
\text { tung } \\
\text { Neid }\end{array}$ & $>$ \\
\hline
\end{tabular}

Übersicht 1: Emotionen

Eine für Politik wichtige Emotion, das objektbezogene ,Haben-Wollen` (Begehren), fehlt in Übersicht 1, da sie unter Positiv-Negativ-Aspekt ambivalent ist, insofern sie einerseits die ,negative' Empfindung von Mangel, andererseits die ,po- 
sitive' Ausrichtung auf Besitzen beinhaltet. Auch Gleichgültigkeit ist in der Taxonomie nicht aufgeführt. Für emotionsbezogenes Framing spielt sie als Non-Emotion dennoch eine Rolle.

Wo sind Emotionen in Frame-Darstellungen zu verorten? Emotionen sind aufs Engste mit Bewertungen verbunden. In der politischen Kommunikation, insbesondere in Wahlkämpfen, gibt es kaum Gegenstände, die unabhängig von Bewertungen - sozusagen objektivistisch - konzeptualisiert werden - seien es politische Situationen und Handlungen, seien es Politiker oder Parteien. Darum enthalten die Elemente in Wert-Funktion oft Begriffe mit einer deontischen Komponente $^{13}$ (z. B. Gefahr durch Staatsverschuldung) oder Begriffe, die eine Wertung implizieren (z. B. niedrigste Arbeitslosigkeit seit Jahren). Es ist der Wertungsgehalt solcher Elemente, der nach übereinstimmender Auffassung der politstrategischen Akteure Emotionen, z. B. Sorge oder Zuversicht, erzeugt. Da es sich bei Emotionen um Empfindungen über Sachverhalte handelt, werden sie in der frameanalytischen Darstellung - entsprechend Busses Einführung von Meta-Elementen in die Frametheorie (Busse 2012, 581 ff.) - auf der Ebene der Attribute als Meta-Attribute und auf der Ebene der Werte als Meta-Werte behandelt. Ihr besonderer Status wird durch Kursivschreibung indiziert. Mit den Sachverhalten, durch die sie ausgelöst werden, sind sie - im Sinne der Barsalou'schen ,constraints ' - verknüpft, und zwar kausal, sodass wir hier immer wieder Kausal-Constraints zwischen jeweiligem Wert und Meta-Wert begegnen.

${ }^{13}$ Den Terminus „deontisch“ hat Hermanns (1986) eingeführt. In politischen Begriffen kommt - anders als etwa im Liebesgedicht - Bewertung nicht subjektiv-individuell daher, sondern als Anspruch auf intersubjektive, gegebenenfalls allgemeine Geltung. Es wäre zu schwach, diesen Bedeutungsaspekt als „Bewertungskomponente“ oder „evaluative Bedeutung“ zu bezeichnen. Es geht um Normen, um überindividuelles Sollen, Müssen und (Nicht-)Dürfen, also um das, was die Logik als „Deontik“ bezeichnet. 


\section{Die CDU-Wahlkampagne 2013. Framing und frame- analytische Modellierung}

\subsection{Ausgangslage und strategische Überlegungen}

Framing-Aufgaben stellen sich bei Wahlkampagnen auf der Makro-Ebene der Gesamtausrichtung, der Meso-Ebene einzelner politikfeld-spezifischer Begriffsnetze und der Mikro-Ebene der Begriffsprägung. Sie betreffen die Modellierung

- politischer Sachlagen,

- des eigenen Partei- und Spitzenkandidatenbildes,

- der politischen Konkurrenz samt Spitzenkandidat,

- des Wählerbildes.

In sachpolitischer Hinsicht werden die Prämissen für chancenreiches Framing neben der generellen programmatischen Ausrichtung - vor allem bei einer Regierungspartei - durch deren praktische Politik und ihre Ergebnisse gesetzt. Bei der Selbstpräsentation, der Gegnermodellierung und dem Wählerbild sind es insbesondere Ergebnisse der Demoskopie, die Spielräume eröffnen oder begrenzen. Teilweise handelt es sich um öffentlich bekannte, im Auftrag von Fernsehanstalten und/oder Presseorganen erhobene Daten, teilweise um nicht-öffentliche, im Hinblick auf den Wahlkampf ermittelte Daten.

Für die Planung der CDU-Kampagne waren - neben den Beliebtheitswerten der Bundeskanzlerin Angela Merkel und ihres SPD-Herausforderers Peer Steinbrück - vor allem folgende Daten wichtig:

- die vorrangige Zuordnung wichtiger politischer Begriffe - und damit potenziell wahlrelevanter Sachverhalte und Werte - zur CDU einerseits und (vor allem) zur SPD andererseits,

- Unterschiedlichkeit in der Relevanz dieser Begriffe für die Wählerschaft.

Im Frühjahr 2013 lagen der CDU folgende Umfrageergebnisse zu parteibezogenen Begriffs-Assoziationen vor: ${ }^{14}$

\footnotetext{
${ }^{14}$ Im Hinblick auf den Wahlkampf interessierten vor allem die Unterschiede zwischen CDU- und SPD-Zuordnung und weniger der (durchaus bemerkenswerte) Umstand, dass viele Befragte keine parteispezifische Zuordnung vornahmen.
} 
Primär der CDU zugeordnet:

$\begin{array}{ll}\begin{array}{l}\text { führungsstark } \\ \text { gutes Regieren }\end{array} & \text { (CDU 47 \%, SPD 14\%) } \\ \begin{array}{l}\text { Wohlstand } \\ \text { Sicherheit }\end{array} & \text { (CDU 33\%, SPD 16\%) } \\ \text { Leistung } & \text { (CDU 37\%, SPD 13\%) } \\ \text { Primär der SPD zugeordnet: } & \text { (CDU 37\%, SPD 16\%) } \\ \text { sozial } & \text { (CDU 33\%, SPD 15\%) } \\ \text { soziale Marktwirtschaft } & \\ \text { Leistungsgerechtigkeit } & \text { (SPD 47\%, CDU 14\%) } \\ & \text { (SPD 33\%, CDU 24\%) } \\ & \text { (SPD 24\%, CDU 18\%) }\end{array}$

Was die Relevanz dieser Begriffe für die Wählerschaft betrifft, war die Lage nicht eindeutig. Einerseits beurteilten in einer aktuellen Umfrage der TNS-InfratestSozialforschung 51,4\% der Befragten ihre eigene wirtschaftliche Lage als gut oder sehr gut, ${ }^{15}$ und auf einer 10er Skala zur Lebenszufriedenheit ordneten sich sogar 71,5 \% bei den Skalenwerten 7-10 (= ziemlich zufrieden - ganz und gar zufrieden) ein. ${ }^{16}$ Die damit zusammenhängenden Begriffe Wohlstand, Sicherheit und Leistung wurden laut Umfrage vorrangig mit der CDU assoziiert. Gleiches galt für gutes Regieren. In allen Umfragen hatte Bundeskanzlerin Merkel stabil die besten Zustimmungswerte. ${ }^{17}$ Laut ARD-Deutschlandtrend waren im Februar $201371 \%$ der Befragten „mit ihrem Wirken [...] zufrieden - ihr bester Wert in der laufenden Legislaturperiode“. ${ }^{18}$ In den Medien wurden Merkel immer wieder die Eigenschaften ,nüchtern' und ,unprätentiös' zugesprochen. Während ihrer Kanzlerschaft war die Arbeitslosigkeit von 11,7 \% im Jahr 2005 auf 6,8 \% im Jahresdurchschnitt 2012 gesunken. Merkel galt in der Euro-Krise, die die Bevölkerung seit 2011 beunruhigte, als Politikerin, die es - bei aller europäischen Orientierung verstand, die deutschen Interessen gegen Begehrlichkeiten der sog. Krisenländer zu wahren.

\footnotetext{
15 http://de.statista.com/statistik/daten/studie/176847/umfrage/beurteilung-eigene-wirtschaftlichelage/.

$16 \mathrm{http} / /$ de.statista.com/statistik/daten/studie/180045/umfrage/gegenwaertige-lebenszufriedenheit/. $\mathrm{Zu}$ einem beinahe identischen Befund kommt eine Umfrage von Allianz und Universität Hohenheim (Der Tagesspiegel 21.03.2013).

${ }^{17}$ Mit Merkel „zufrieden“/,sehr zufrieden“: 71 \% (ARD-DeutschlandTREND Februar 2013).

$18 \mathrm{http}: / /$ www.infratest-dimap.de/umfragen-analysen/bundesweit/ard-deutschlandtrend/2013/februar/.
} 
Andererseits war in der Zeit der schwarz-gelben Koalition 2009-2013 das primär der SPD zugeordnete Thema „soziale Gerechtigkeit“ vor allem unter dem Aspekt diesbezüglicher Defizite zum weithin beherrschenden politischen Thema in den Medien geworden. Die Assoziation der Begriffe ,sozial' und ,gerecht' mit der SPD war - im Vergleich mit der CDU - laut oben zitierter Umfrage so eng, dass selbst die Begriffe Marktwirtschaft und Leistung, die isoliert weit mehr mit der CDU assoziiert werden, in den Zusammensetzungen soziale Marktwirtschaft und Leistungsgerechtigkeit primär der SPD zugeordnet wurden. In einer am 12. Februar 2013 veröffentlichten repräsentativen Umfrage des Instituts für Demoskopie Allensbach zum Gerechtigkeitsbegriff heißt es im Fazit:

Die große Mehrheit der Bürger hat einen umfassenden, anspruchsvollen Gerechtigkeitsbegriff, der Chancen- und Leistungsgerechtigkeit genauso umfasst wie Familien- und Generationengerechtigkeit sowie Verteilungsgerechtigkeit. In allen Gerechtigkeitsdimensionen sehen die Bürger erheblichen Handlungsbedarf. [...] Entsprechend ist die Politik mit hohen Erwartungen konfrontiert. ${ }^{19}$

Die von der SPD geforderten Konkretisierungen im Bereich der Verteilungsgerechtigkeit wie Mindestlohn und Maßnahmen zur Schaffung von Steuergerechtigkeit fanden mit je $76 \%$ die höchsten Zustimmungswerte (vgl. ebenda).

Demgegenüber sehen zum gleichen Zeitpunkt die Werte für den SPD-Kanzlerkandidaten Steinbrück schlecht aus: Laut ARD-Deutschlandtrend würden sich bei einer Kanzler-Direktwahl im Februar 2013 nur „28 Prozent für Peer Steinbrück entscheiden“ gegenüber „59 Prozent für die Amtsinhaberin“. ${ }^{20}$ Immer wieder wurde ein Widerspruch zwischen dem sich abzeichnenden ,linken“ Wahlprogramm der SPD und dem in der Vergangenheit als wirtschaftsnah und marktwirtschaftlich orientiert geltenden Kanzlerkandidaten thematisiert. Monatelang hatte es Schlagzeilen und kritische Berichterstattung wegen sehr hoher Honorare für Steinbrück als Redner im Kontext ,der Wirtschaft‘ gegeben. Dazu kamen Nichtigkeiten, die vor allem von der Boulevardpresse skandalisiert wurden. Steinbrücks Reaktionen galten vielfach als ungeschickt und/oder dünnhäutig.

${ }^{19}$ Vgl. http://www.insm.de/insm/kampagne/gerechtigkeit/was-denkt-deutschland-ueber-gerechtigkeit.html; letzter Zugriff: 6. Juli 2015.

$20 \mathrm{http} / /$ www.infratest-dimap.de/umfragen-analysen/bundesweit/ard-deutschlandtrend/2013/februar/; letzter Zugriff: 6. Juli 2015. 


\subsection{Framing und Frames auf der Makroebene}

Da Kampagnen-Akteure vor der Aufgabe stehen, möglichst viele Wähler/innen aus der Menge der für die CDU zugänglichen Wahlberechtigten zu gewinnen, ist es vorrangig, sich zunächst ein ungeschminktes Bild von deren

- Einschätzung der eigenen Lage,

- politischem Kenntnisniveau,

- politischen Themen-Prioritäten,

- Zustimmung zum politischen Spitzenpersonal,

- (normativen) Ansprüchen an Politiker/innen

zu machen. Das Bild des Wählers/der Wählerin (s. o.) beruht auf demoskopischen Daten und auf alltagsweltlichem, in Kommunikation und Medienerfahrungen aggregiertem Wissen.

\begin{tabular}{|c|c|c|}
\hline \multicolumn{3}{|c|}{ Kategorie: (CDU-relevante) Wahlberechtigte } \\
\hline ATTRIBUT & WERT & Meta-Attribut EMOTION \\
\hline SELBSTEINSCHÄTZUNG & $\begin{array}{l}\text { gute wirtschaftliche } \\
\text { Lage }\end{array}$ & $\rightarrow$ Zufriedenheit, Freude \\
\hline $\begin{array}{l}\text { POLITISCHE KENNT- } \\
\text { NISSE }\end{array}$ & ungenau, defizitär & \\
\hline THEMENPRIORITÄTEN & $\begin{array}{l}\text { Wirtschaft/Arbeits- } \\
\text { markt } \\
\text { Eurokrise } \\
\text { Soziale Gerechtigkeit }\end{array}$ & $\begin{aligned} \longrightarrow & \text { Zufriedenheit, Zuversicht } \\
\longrightarrow & \text { Ärger, Sorge } \\
\longrightarrow & \text { Arger, Begehren, Mitleid, } \\
& \text { Gleichgültigkeit }\end{aligned}$ \\
\hline KANZLERPRIORITÄT & Merkel & $\rightarrow$ Vertrauen \\
\hline $\begin{array}{l}\text { ANSPRÜCHE AN POLITI- } \\
\text { KER }\end{array}$ & $\begin{array}{l}\text { politikrelevante ,Tu- } \\
\text { genden' (s. Subframe } \\
\text {,Tugenden') }\end{array}$ & \\
\hline
\end{tabular}

Übersicht 2: Wähler-Frame

In der frameanalytischen Darstellung (Übersicht 2: Wähler-Frame) bilden die genannten Aspekte die Attribute (im Barsalou'schen Sinne). Deren Ausprägungen (Werte) interessieren nicht nur in inhaltlicher Hinsicht, sondern auch unter dem 
Gesichtspunkt, welche Emotionen sie aus Sicht der Kampagnenakteure typischerweise auslösen. Die Emotionen sind in einer Spalte für die Werte des MetaAttributes EMOTION notiert. Der angenommene Auslöseeffekt wird als KausalConstraint durch einen Pfeil symbolisiert.

Die in der Wählerschaft vorherrschenden normativen Ansprüche an Politiker betreffen etliche Aspekte persönlichen Verhaltens. Sie bilden eine Art Tugendkatalog, dessen Bestandteile einem Common Sense weit über den Bereich der Politik hinaus entsprechen und der wegen seines Umfangs als Subframe des WählerFrames präsentiert wird (Übersicht 3).

\begin{tabular}{|c|c|c|}
\hline \multicolumn{3}{|c|}{ Kategorie: (politikrelevante) Tugenden } \\
\hline Attribut & Wert & $\begin{array}{c}\text { Meta-Attribut } \\
\text { EMOTION }\end{array}$ \\
\hline KÖNNEN & sachkundig, durchsetzungsfähig & \\
\hline MORAL & glaubwürdig, skandalfrei & \\
\hline GRUNDHALTUNG & $\begin{array}{l}\text { ausgeglichen (= weder zu rational } \\
\text { noch zu emotional), nicht abgeho- } \\
\text { ben, unprätentiös }\end{array}$ & $\begin{array}{l}\text { Achtung, } \\
\text { Wohlwollen }\end{array}$ \\
\hline ARBEITSHALTUNG & pflichtbewusst, leistungsorientiert & \\
\hline KOMMUNIKATION & $\begin{array}{l}\text { dialogorientiert, verständlich, } \\
\text { rhetorisch, fesselnd }\end{array}$ & \\
\hline
\end{tabular}

Übersicht 3: Subframe (politikrelevante) Tugenden

Wie sehr dies für die Bilder von Merkel und Steinbrück in der Wählerschaft prägend ist, wird in den Frames zu Kanzlerin (Übersicht 5) und Kanzlerkandidat (Übersicht 6) deutlich. Der fließende Übergang zwischen allgemeinen ,Tugenden “ und den Verhaltensansprüchen an Politiker(innen) lässt es möglich erscheinen, per Framing eine so große Nähe zwischen Wählerschaft und Kandidat bzw. Kandidatin zu konstruieren, dass sie Wähler(innen) zur Identifikation mit dem Kandidaten/der Kandidatin motivieren kann. (Dazu, inwieweit das 2013 im Hinblick auf das Verhältnis zwischen Deutschland/den Deutschen und Angela Merkel versucht wurde, Näheres in Abschnitt 4.2.2.2)

\subsubsection{Vor Beginn der Kampagne: Ausgangsframes}

Drei Politikfelder wurde als kampagnenrelevant identifiziert: Soziale Gerechtigkeit, Eurokrise und Wirtschaft/Arbeitsmarkt (s. Übersicht 4). Anders als in der 
Kommunikation mit politisch stark Interessierten, die die Thematisierung des gesamten Spektrums der Topik politischen Handeln (s. Abschnitt 1) erforderlich macht, sah man das Interesse des politisch nur mäßig interessierten Durchschnittswählers bei allen Themen primär auf die - mehr oder weniger aktuelle Lage und auf die Verantwortlichkeit dafür gerichtet.

Bei der sozialen Gerechtigkeit wurden, wie die oben erwähnten Umfragen zeigen, Defizite in der Verantwortung der Regierung und/oder des Wirtschaftssystem gesehen. Dementsprechend herrschten kritische Einstellungen und negative Emotionen vor - vom Mitgefühl für Benachteiligte bis zum Neid auf Bessergestellte. In der Öffentlichkeit stark präsent waren polarisierungsträchtige Forderungen von SPD, Grünen und der Linken nach mehr sozialer Gerechtigkeit, z. B. Mindestlohn, mit unterschiedlichem Emotionspotenzial von Hoffnung bis Skepsis.

Die Eurokrise wurde wahrgenommen als Staatsverschuldung der Krisenländer, die den Euro (,unsere Währung') gefährdet. Das bereitete Sorge, andererseits sah man ein entschlossenes Gegensteuern insbesondere der deutschen Kanzlerin, indem Hilfen für die betroffenen Staaten nur bei Haushaltsdisziplin (Sparen) und Strukturreformen gewährt wurden. Dem wurde teils mit Zuversicht, teils mit Skepsis begegnet.

In der Bevölkerung dominierte der Eindruck von gut laufender Wirtschaft (Exportrekorde) und relativ geringer Arbeitslosigkeit. Üblicherweise wird das vom Gros der Wähler nicht zuletzt der Wirtschafts- und Arbeitsmarkt-Politik der Regierung zugeschrieben und ist emotional positiv besetzt - von der Zufriedenheit mit der eigene Situation bis zum Vertrauen in die Kanzlerin.

Die drei in ihrer Wertigkeit noch nicht entschiedenen Themen bildeten ein Frame-Cluster mit den Attributen LAGE und VERANTWORTLICHKEIT. Sie gehören als strukturelle Invarianten zusammen. Während beim Thema soziale Gerechtigkeit die REAKTION der Oppositionsparteien auf LAGE und VERANTWORTLICHKEIT (Forderung nach mehr sozialer Gerechtigkeit, insbesondere nach flächendeckendem Mindestlohn) als in der Wählerschaft allgemein bekannt eingeschätzt wurde und daher in den Frame aufgenommen wurde, galt dies für die anderen Themen nicht. 
Das öffentliche Bild der Bundeskanzlerin Angela Merkel ist - neben den sachpolitischen Aspekten (s. Übersicht 4) und dem Wissen über ihre hohen Zustimmungswerte - geprägt durch die Wahrnehmung ihres politischen Stils, wobei für die potenzielle CDU-Wählerschaft nach Auffassung der Kampagnenakteure vor allem die im Ausgangsframe Merkel (Übersicht 5) notierten Qualitäten und deren emotionale Auswirkungen relevant waren. Bemerkenswert ist die erhebliche Übereinstimmung mit den in Übersicht 3 dargestellten ,Tugendkatalog،

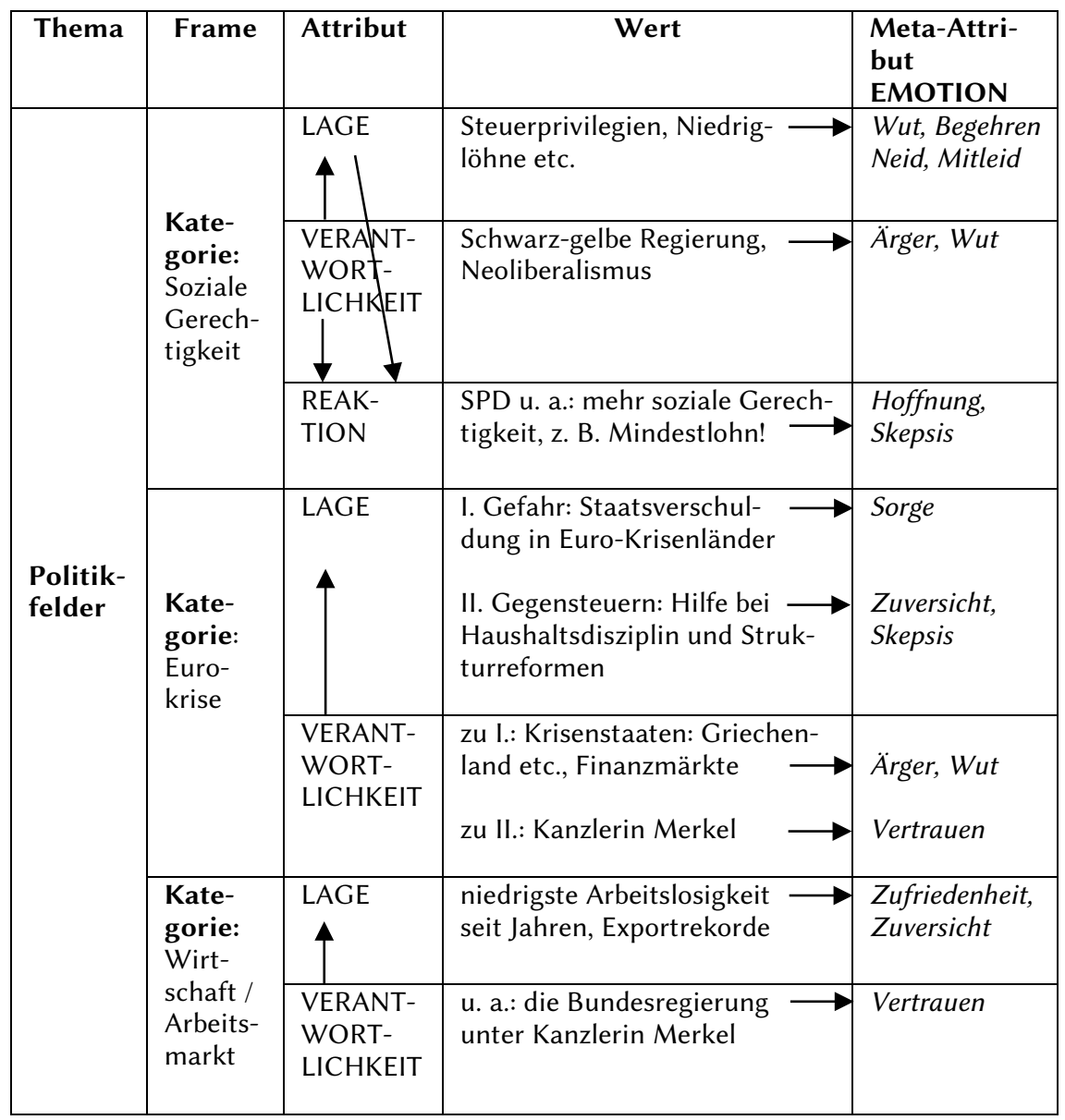

Übersicht 4: Ausgangs-Framecluster, Politikfelder Legende: Pfeil: Kausalrelation (Ursache/Grund $\longrightarrow$ Wirkung/Folge) 


\begin{tabular}{|l|l|l|}
\hline \multicolumn{2}{|c|}{ Kategorie: Bundeskanzlerin Merkel } \\
\hline \multicolumn{1}{|c|}{ Attribut } & \multicolumn{1}{|c|}{ Wert } & \multicolumn{1}{|c|}{$\begin{array}{c}\text { Meta-Attribut } \\
\text { EMOTION }\end{array}$} \\
\hline AKTUELLER STATUS & $\begin{array}{l}\text { Bundeskanzlerin (CDU), } \\
\text { hohe Zustimmungswerte } \\
\text { Vertrauen }\end{array}$ \\
\hline KÖNNEN & $\begin{array}{l}\text { kompetent, durchsetzungsfähig } \\
\text { erfolgreich in Wirtschafts-, } \\
\text { Arbeitsmarkt- u. Eurokrisenpoli- } \\
\text { tik }\end{array}$ & $\begin{array}{l}\text { Achtung, } \\
\text { Vertrauen }\end{array}$ \\
\hline MORAL & \begin{tabular}{l} 
skandalfrei, glaubwürdig \\
\cline { 3 - 3 }
\end{tabular} & $\begin{array}{l}\text { Vertrauen, } \\
\text { Sympathie }\end{array}$ \\
\hline TEMPERAMENT & $\begin{array}{l}\text { gelassen, rational ohne gefühllos } \\
\text { zu wirken }\end{array}$ & Sympathie \\
\hline ARBEITSHALTUNG & $\begin{array}{l}\text { pflichtbewusst, leistungs- } \\
\text { orientiert }\end{array}$ & $\begin{array}{l}\text { Achtung, } \\
\text { Vertrauen }\end{array}$ \\
\cline { 3 - 3 } & $\begin{array}{l}\text { unprätentiös, beherrscht, } \\
\text { seriös }\end{array}$ & $\begin{array}{l}\text { Sympathie, } \\
\text { Vertrauen }\end{array}$ \\
\hline AUSSTRAHLUNG & &
\end{tabular}

$$
\begin{gathered}
\text { Übersicht 5: Ausgangsframe Merkel } \\
\text { Legende: Pfeil: Kausalrelation (Ursache/Grund } \longrightarrow \text { Wirkung/Folge) }
\end{gathered}
$$

An dieser Stelle liegt die Frage nach einem eigenen CDU-Frame nahe. Darauf wird verzichtet, da in der Kampagne kein gegenüber der Spitzenkandidatin und Bundeskanzlerin Angela Merkel auch nur annähernd eigenständiges Parteibild entwickelt wurde. Das hatte gewichtige Gründe: Auch die CDU hatte - trotz ihres Vorsprungs auf Bundesebene vor den anderen Parteien - mit dem weit verbreiteten Misstrauen gegenüber Parteien zu kämpfen. Ihre Zustimmungswerte als Partei lagen seit langer Zeit weit hinter denen ihrer Vorsitzenden und Bundeskanzlerin Angela Merkel zurück. Auch wusste man, dass das Bild der CDU selbst bei ihren potenziellen Wählern - nicht ganz einheitlich war und dass bei Betonung des Partei-Charakters auch das nicht spannungsfreie Verhältnis zur CSU Thema sein würde. Die Kampagne sollte darauf hinauslaufen, das Bild der CDU ganz mit dem Bild von Angela Merkel zu verschmelzen - nach dem Prinzip: Mit optimalem Merkel-Framing framen wir die CDU gleich mit. Vor diesem Hintergrund wird auf die Modellierung des Akteurswissens über das CDU-Bild vor Beginn der Kampagne verzichtet. 
Auch ein eigener SPD-Frame fehlt - allerdings nicht wegen Verschmelzung mit dem Steinbrück-Frame, sondern weil die Aspekte der SPD-Politik, die die Kampagnenakteure als relevant für potenzielle CDU-Wähler annahmen, in den Politikfelder-Frames (Übersichten 4 und 8) enthalten sind.

Die im Verhältnis zu Merkel bemerkenswert geringen Zustimmungswerte für den - zuvor als Finanzpolitiker durchaus beliebten - Kanzlerkandidaten Peer Steinbrück führten die Kampagnenakteure der CDU auf sein Profil zurück, das dem ,Tugendkatalog ' für Politiker in etlichen Punkten nicht entspricht (Übersicht 6).

\begin{tabular}{|c|c|c|}
\hline \multicolumn{3}{|c|}{ Kategorie: SPD-Kanzlerkandidat Steinbrück } \\
\hline Attribut & Wert & $\begin{array}{c}\text { Meta-Attribut } \\
\text { EMOTION }\end{array}$ \\
\hline $\begin{array}{l}\text { AKTUELLER } \\
\text { STATUS }\end{array}$ & $\begin{array}{l}\text { Kanzlerkandidat (SPD) } \\
\text { affärengeplagt, niedrige } \\
\text { Zustimmungswerte }\end{array}$ & $\longrightarrow$ Misstrauen, Antipathie \\
\hline KÖNNEN & $\begin{array}{l}\text { finanz-/wirtschaftspolitisch } \\
\text { kompetent, } \\
\text { rhetorisch brillant }\end{array}$ & $\begin{array}{l}\longrightarrow \text { Vertrauen (sektoral) } \\
\longrightarrow \text { Achtung }\end{array}$ \\
\hline MORAL & $\begin{array}{l}\text {,Absahner', } \\
\text { sozialpolitisch wenig glaub- } \\
\text { würdig }\end{array}$ & $\begin{array}{l}\longrightarrow \text { Antipathie } \\
\longrightarrow \text { Misstrauen (sektoral) }\end{array}$ \\
\hline $\begin{array}{l}\text { TEMPERA- } \\
\text { MENT }\end{array}$ & $\begin{array}{l}\text { extrovertiert, } \\
\text { gelegentlich unkontrolliert }\end{array}$ & $\begin{array}{l}\longrightarrow \text { Sympathie, Skepsis } \\
\longrightarrow \text { Unmut }\end{array}$ \\
\hline $\begin{array}{l}\text { ARBEITSHAL- } \\
\text { TUNG }\end{array}$ & leistungsorientiert & $\longrightarrow$ Achtung \\
\hline $\begin{array}{l}\text { AUSSTRAH- } \\
\text { LUNG }\end{array}$ & $\begin{array}{l}\text { Anflüge von Arroganz, } \\
\text {,machohaft }\end{array}$ & $\longrightarrow$ Antipathie \\
\hline
\end{tabular}

$$
\begin{gathered}
\text { Übersicht 6: Ausgangsframe Steinbrück } \\
\text { Legende: Pfeil: Kausalrelation (Ursache/Grund } \longrightarrow \text { Wirkung/Folge) }
\end{gathered}
$$

\subsubsection{Strategisches Framing und Zielframes}

Strategisches Framing hat dann eine Chance, wenn die strategische Hauptausrichtung einer Kampagne erfolgversprechend ist. Angesichts der guten Wirtschafts- und Arbeitsmarktdaten und der hohen Zufriedenheitswerte in der Bevölkerung sowohl mit der eigenen persönlichen Situation als auch mit der Arbeit der 
Kanzlerin entschied man sich in der CDU zu einer feel-good-Kampagne. Von Seiten der SPD sah man eine Wut-Sorge-Mitleid-Kampagne oder zumindest einen alarmistischen Wahlkampf wegen ,sozialer Ungerechtigkeiten' und Gefährdung des ,Auseinander-Driftens der Gesellschaft‘ auf sich zukommen, zu dem allerdings der Kanzlerkandidat nicht so recht passte. Für die Kampagne der CDU hieß das, vor allem

- die Stärken der CDU und der Kanzlerin hervorzuheben und den Ansehensvorsprung Merkels im Spitzenkandidaten-Wettbewerb auszubauen oder zumindest zu halten,

- die sachpolitischen Schwächen der CDU im Feld, soziale Gerechtigkeit‘ zu beheben oder zumindest zu minimieren oder zu neutralisieren,

- vor diesem Hintergrund Deutschland nicht als gesellschaftlich spaltungsbedroht, sondern als funktionierende ,Gemeinschaft ${ }^{\prime} \mathrm{zu}$ konzeptualisieren,

- Polarisierung zu vermeiden und ausschließlich moderate Emotionen zu mobilisieren.

Damit war für Framing die Aufgabe gestellt, die in den Ausgangsframes erfassten Vorstellungen im Sinne des Wahlzieles und der Hauptausrichtung der Kampagne zu modifizieren bzw. zu optimieren und als verbessertes Bild der CDU-Politik und der Kanzlerin in den Köpfen möglichst vieler Wahlberechtigter zu verankern. Die so erzeugten Vorstellungen werden als Zielframes präsentiert (Übersicht 7 und 8).

\subsubsection{Spitzenkandidaten-Konkurrenz}

Im Hinblick auf die Spitzenkandidaten-Konkurrenz lag es nahe, sich an eine bewährte Faustregel zu halten: „Werte bei starkem eigenem Kandidaten (hier: Kandidatin) einen schwachen Gegenkandidaten nicht durch starke Beachtung auf!“ Die CDU und insbesondere Merkel selbst hielten sich strikt daran. Sie folgten dem Kalkül: Solange der Kanzlerkandidat der SPD sich durch skandalisierungfähige Fehler selbst in Negativschlagzeilen bringt und es nicht vermag, die Glaubwürdigkeitslücke zwischen parteioffizieller Propagierung ,sozialer Gerechtigkeit und dem Ruch des teuren wirtschaftsfreundlichen Vortragsredners zu schließen, thematisieren wir ihn von uns aus nicht. Daher wurde Framing zur Korrektur des im Ausgangsframe Steinbrück (Übersicht 6) manifestierten Profils erst gar nicht in Angriff genommen. In den Wahlkampfmedien der CDU kommt er in Wort und 
Bild nicht vor. Die Kanzlerin nimmt bis zum TV-Duell seinen Namen nicht in den Mund. Steinbrücks Attacken, auch heftige, spielt sie ostentativ herunter. Wo sie auf ihn angesprochen wird, erinnert sie an die gute Kooperation in der Großen Koalition. $^{21}$

\subsubsection{Kanzlerinnen-Framing}

Im Bild der Kanzlerin in der Öffentlichkeit finden sich, wie im Ausgangsframe Merkel (Übersicht 5) deutlich wird - anders als bei Steinbrück - etliche der erwünschten ,Tugenden'. Es galt in der Wahlkampagne, diese Positiv-Werte zu bestärken und dort, wo Defizite oder ,blinde Flecken ' waren, sie möglichst zu beseitigen. So boten die Schwerpunkte Wirtschaft/Arbeitsmarkt und Eurokrise die Möglichkeit einer permanenten Bestärkung der Positiv-Werte für Merkel insbesondere beim Attribut KÖNNEN.

Auch visuelles Framing wurde genutzt, um Eigenschaften der Kanzlerin zu unterstreichen, die schon im Ausgangsframe positive Werte aufwiesen: So signalisiert Merkels Outfit in den verschiedensten Bild-Medien Seriosität, und im TVSpot der CDU demonstriert Merkel optisch eindringlich Leistungsbereitschaft und Pflichtbewusstsein. Man sieht eine Frau, deren Falten von der Arbeit für Deutschland zeugen, über die sie spricht, und die das in einem wenig ansprechenden nüchternen Raum tut, wie ihn Millionen aus ihrem Arbeitsalltag kennen insgesamt eine Situation, mit der viele Menschen sich identifizieren können.

Letzteres berührt eine Dimension, in der Merkels Ausgangsframe ohne Ausprägung war und wo es Einiges zu verbessern galt: Kommunikation, Gemeinsamkeit und Nähe zu ,normalen Menschen‘. Merkels weithin hoch geschätzte Eigenschaft, unprätentiös zu sein, erleichterte Framing in Richtung ,Normalisierung der Bundeskanzlerin. Das geschah primär in Texten, aber auch in Bildern. Auf Merkels eigens für die Wahlkampagne eingerichteter sog. ,Homepage“ und in der damit text- und bild-identischen, massenhaft verteilten Merkel-Broschüre wird einerseits die entscheidungsstarke, durchsetzungsfähige Politikerin nicht verleugnet (,ich will...“; „ich entscheide...“), andererseits ist sie, wenn es um Privates geht, eine ,wie du und ich': „Ich koche sehr gern, am liebsten Rouladen und Kartoffelsuppe.“ Ihr Ehemann, ein „Konditorsohn“ beschwert sich, „auf dem Kuchen

\footnotetext{
${ }^{21} \mathrm{Zu}$ Einzelheiten vgl. Klein (2013, 197 f.)
} 
sind immer zu wenig Streusel." Dies blieb der einzige textliche Hinweis auf Weiblichkeit im traditionellen Sinne. Eine stärkere Signalisierung von Geschlechtsspezifik barg die Gefahr, das Identifikationspotenzial zum einen für männliche Wähler, zum anderen für Anhänger/innen eines ,modernen` Frauenbildes zu schmälern.

Wichtiger als die Präsentation in kampagnenspezifischen Schriftmedien dürften für den Eindruck, sie sei - auch - ,eine von uns', sprachstilistische Mittel in der Standard-Wahlrede gewesen sein, mit der sie über die Plätze und durch die Säle der Republik zog. Darin stellt sie ihre Politik in einfachster Sprache dar und thematisiert persönliches Erleben und Gefühle, um politische Zusammenhänge nachvollziehbar zu machen, etwa zum Thema Europa - so am 16.08.2013 in Waren an der Müritz:

Und als ich auch mal'n bisschen verzweifelt über die Streitereien der Regierungschefs war, hab' ich mich gefragt: Was machst Du da eigentlich die vielen Stunden? Da hab ich mir mal überlegt, worüber wir uns nicht streiten in Europa: Wir streiten uns nicht darüber, dass wir Demokratie haben. Wir streiten uns nicht darüber, dass wir Pressefreiheit haben. Wir streiten uns nicht darüber, dass wir Meinungsfreiheit haben, dass wir Reisefreiheit haben. Und wir streiten uns auch nicht darüber, dass wir Religionsfreiheit haben. Das alles ist selbstverständlich in allen 28 Ländern Europas. Und diese Gemeinsamkeit, worüber wir uns nicht streiten, die ist unglaublich viel wert, wenn Sie mal daran denken, was in Ägypten, in Syrien und in anderen Ländern los ist. Das ist unser Europa, und das ist unsere Stärke. ${ }^{22}$

Alltagssprachlich und alltagslogisch rechtfertigt sie in dieser Rede auch ihren konfrontationsarmen Wahlkampfstil: „Ich hab' meine Zeit heute im Wesentlichen darauf verwandt, Ihnen zu sagen, was wir wollen. Ich hab' keine Lust den ganzen Wahlkampf damit zu verbringen zu erklären, was die anderen alles falsch machen." (Dafür erhält sie stets besonders starken Applaus, vgl. ebenda.)

Merkel beendet die Standardrede jeweils mit ausgesprochen höflichem Dank für die Aufmerksamkeit und für den Besuch der Veranstaltung, nachdem sie zuvor nicht nur auf die Wichtigkeit des Wählens, sondern auch - geschickt verbunden mit einer unaufdringlichen Empfehlung, der CDU die Stimme zu geben - auf die Freiheit des Wahlakts hinweist. Da wird vermittelt, dass das Volk der Souverän ist.

${ }^{22}$ www.youtube.com/watch?v=7--kmuylfLQ; letzter Zugriff am 07.06.2015. 
Die Kommunikatorin Merkel ist auch Gegenstand visuellen Framings: Zwei der drei Merkel-Großplakate zeigen sie aufmerksam und freundlich im Gespräch. ${ }^{23}$ Auch die Fotos in Merkel-Homepage und -Broschüre betonen lockere Kommunikativität stärker als Amtswürde.

Das alles begünstigt das Motiv der Gemeinsamkeit zwischen Merkel, Volk und CDU. Es handelt sich primär um eine Leistungsgemeinschaft, wie der Kampagnen-Claim ausweist: Gemeinsam erfolgreich. Der Claim der Obama-Kampagne Yes, we can klingt an - hier nicht mit Blick auf das, was man verändern will, sondern auf das, was man mit der Kanzlerin erreicht hat und was weitergeführt werden soll. Oft erweitert um den Zusatz Für Deutschland ziert der Slogan alle Wahlkampfmedien der CDU. Im Kontrast zur SPD-Kampagne, für die sich schon früh abzeichnete, dass sie ein Auseinander-Driften der Gesellschaft behaupten würde, wurde der Zusammenhalt betont, z. B. im ersten Abschnitt des Wahlprogramms. Gemeinschaft ist nicht als Kollektiv verstanden. An markanten Stellen wird Individualität herausgestellt: Die Menschen sind ja alle ganz verschieden (Merkels Standardrede), jeder einzelne von Ihnen (ebenda), weil jeder zählt (Großplakat mit Merkel). Visuell werden die Angehörigen dieser Gemeinschaft als aktiv und fröhlich geframet: Die erste große Plakatwelle der CDU präsentiert gut gelaunte Junge und Alte, Frauen und Männer zu Hause, bei der Arbeit oder anderswo in Situationen voller Lebendigkeit als Repräsentanten der gemeinsam Erfolgreichen (vgl. ebenda). Es geht dabei um ein (vor allem wirtschaftlich) starkes Deutschland, wie das (als „Regierungsprogramm 2013-2017“ bezeichnete) Wahlprogramm von CDU und CSU sowie etliche andere Wahlkampftexte in hoher Frequenz der Epitheta stark, stabil, erfolgreich, gut und sicher ausweisen.

Die nationale Dimension von Gemeinschaft deutet Merkel mit den Farben Schwarz, Rot und Gold ihrer Halskette im TV-Duell auch visuell an. Dort richtet Merkel einen vielzitierten Satz an die Zuschauer, der Nähe des Volkes zu ihr behauptet: „Sie kennen mich.“

Sämtliche Framing-Züge zur Optimierung des Merkel-Bildes bei potenziellen CDU-Wählern zielte auf Verknüpfung mit positiven Emotionen, und zwar ausschließlich moderaten Emotionen, wie man sie schon im Ausgangsframe Merkel

\footnotetext{
${ }^{23}$ Vgl. https://www.cdu.de/plakate; letzter Zugriff: 7. Juli 2015.
} 
findet. Eine solche Emotionsstrategie führt zwar nicht zu massenhafter Identifikation begeisterter Anhänger mit ihr, doch öffnet sie Zugangschancen zu denen, die Polarisierung eher abstoßend finden.

\begin{tabular}{|c|c|c|}
\hline \multicolumn{3}{|c|}{ Kategorie: Angela Merkel } \\
\hline Attribut & Wert & $\begin{array}{c}\text { Meta-Attribut } \\
\text { EMOTION }\end{array}$ \\
\hline AKTUELLER STATUS & $\begin{array}{l}\text { Bundeskanzlerin (CDU); hohe Zu- } \\
\text { stimmungswerte }\end{array}$ & $\begin{array}{l}\longrightarrow \text { Achtung, Vertrauen, } \\
\text { Zuversicht }\end{array}$ \\
\hline KÖNNEN & $\begin{array}{l}\text { sachkundig; durchsetzungsfähig; } \\
\text { erfolgreich in Wirtschafts-, Ar- } \\
\text { beitsmarkt- und Eurokrisenpolitik }\end{array}$ & $\longrightarrow$ Achtung, Vertrauen \\
\hline MORAL & skandalfrei; glaubwürdig & $\longrightarrow$ Vertrauen, Sympathie \\
\hline TEMPERAMENT & $\begin{array}{l}\text { gelassen; rational ohne gefühllos } \\
\text { zu sein }\end{array}$ & $\begin{array}{l}\longrightarrow \text { Wohlwollen, Sympa- } \\
\text { thie }\end{array}$ \\
\hline ARBEITSHALTUNG & $\begin{array}{l}\text { pflichtbewusst, leistungsorientiert } \\
\text { (TV-Spot: Falten als Zeichen } \\
\text { harter Arbeit) }\end{array}$ & $\longrightarrow$ Achtung, Vertrauen \\
\hline AUSSTRAHLUNG & $\begin{array}{l}\text { unprätentiös, beherrscht } \\
\text { (Outfit: seriös) }\end{array}$ & $\begin{array}{l}\text { Wohlwollen, Ver- } \\
\text { trauen }\end{array}$ \\
\hline WÄHLERNÄHE & $\begin{array}{l}\text {,wie du und ich': kochen, ba- } \\
\text { cken; Wendung an die Zu- } \\
\text { schauer im TV-Duell: „Sie ken- } \\
\text { nen mich.“ }\end{array}$ & $\longrightarrow$ Sympathie \\
\hline $\begin{array}{l}\text { KOMMU- } \\
\text { NIKATION }\end{array}$ & $\begin{array}{l}\text { einfache Sprache mit persönli- } \\
\text { chen Elementen; Wähler/-in- } \\
\text { nen als Souverän ansprechen } \\
\text { (Plakate: Merkel im Gespräch) }\end{array}$ & $\rightarrow$ Sympathie, Ver- \\
\hline $\begin{array}{l}\text { GEMEINSCHAFTS- } \\
\text { BILD }\end{array}$ & $\begin{array}{l}\text { „gemeinsam erfolgreich“: Zu- } \\
\text { sammenhalt zuversichtlicher, } \\
\text { leistungsorientierter Indivi- } \\
\text { duen;starkes Deutschland als } \\
\text { „Stabilitätsanker“ in Europa } \\
\text { (Halskette beim TV-Duell in } \\
\text { Nationalfarben) }\end{array}$ & $\begin{array}{l}\longrightarrow \text { Vertrauen, Zuver- } \\
\text { sicht, Stolz }\end{array}$ \\
\hline
\end{tabular}

Legende:

Pfeil:

Fettdruck:

Übersicht 7: Zielframe Merkel

Unterstreichung: im Ausgangsframe vorhanden und in der Kampagne deutlich gestärkt

Klammer: visuelles Framing 
So ergibt sich ein Zielframe Merkel (Übersicht 7), der einige gegenüber dem Ausgangsframe neue Attribute enthält. Von den Merkel'schen Charakteristika, die der Ausgangsframe enthält, sind in der Kampagne mehrere deutlich bestärkt worden.

\subsubsection{Politikfelder-Framing}

Vor Beginn von Wahlkämpfen pflegt sich die Wählerschaft - vor allem im Hinblick auf regierende Parteien - primär für die aktuelle Politik zu interessieren. Doch mit Eintreten in die Kampagnenphase wird auch regierenden Parteien zunehmend die Frage nach künftiger Politik gestellt. Das beantworten Parteien mit ihren Wahlprogrammen und den daran orientierten Wahlkampftexten - von Reden und Interview-Statements über Slogans bis zu Argumentationshilfe für Kandidaten. Darum ist der Zielframe Politikfelder (Übersicht 8) gegenüber dem Ausgangsframe (Übersicht 4) um das Attribut PROGRAMM erweitert.

In den Medien dominierten schon weit vor Beginn des Bundestagswahlkampfs 2013 die Themen ,soziale Gerechtigkeit' und ,Eurokrise‘. In Meinungsumfragen wurde soziale Gerechtigkeit als sehr wichtig eingestuft. Der Begriff war primär mit der SPD verbunden, während der CDU/CSU/FDP-Regierung hier Defizite angekreidet wurden. Eurokrise war ein seit mehreren Jahren aktuelles Gefahrenthema. Wirtschaft und Arbeitsmarkt standen dagegen in den Medien zurück.

Angesichts dieser Situation war die entscheidende Frage: Was interessiert die für die CDU relevante Wählerschaft im Hinblick auf die Wahlentscheidung vorrangig? Allen medialen Mutmaßungen über eine entscheidende Bedeutung des Themas ,soziale Gerechtigkeit‘ zum Trotz entschieden sich die Akteure der Union für das Clinton-Motto: „It's the economy“, stupid. Strategisches Framing setzte dabei an, dass in der Bevölkerung Zufriedenheit mit der eigenen wirtschaftlichen Lage deutlich überwog, ein Phänomen, das mit der Wirtschafts-, Finanz- und Arbeitsmarktpolitik eng verbunden ist. Die im Ausgangsframe noch ungewichteten Politikfelder wurden in eine Relevanzordnung gebracht. Gegen den medialen Trend wurde der Themenbereich Wirtschaft (Deutschlands Wohlstand sichern), Finanzen (solide Finanzen; Schuldenbremse) und Arbeitsmarkt (Rekordbeschäftigung) unter dem Label ,starkes Deutschlands“ an die Spitze der Kampagnenthemen, eng verknüpft mit Angela Merkel und ihrer Politik in der Eurokrise (stabiler Euro; Deutschland als Stabilitätsanker), gesetzt. Die Akteure gingen davon aus, 
dass gerade auf diesem Politikfeld das Gros der für die CDU relevante Wählerinnen und Wähler eher ein ,Weiter so ' wünschte. Daher bleibt das Programm hier vage. Am entschiedensten ist immer wieder die Ablehnung von Steuererhöhungen formuliert, dem Begriff, unter dem die CDU die von Rot-Grün geforderten Änderungen der Steuergesetzgebung zusammenfasste.

Mit der Eurokrise als zweitem Politikfeld zielte man auf das Sicherheitsbedürfnis der Wählerinnen und Wähler - auch das letztlich von der Clinton'schen Kampagnenmaxime geleitet. Das Framing zur Eurokrisenpolitik wurde von Angela Merkel selbst geprägt. Sie kommunizierte ein Begriffsnetz, das sie situations- und adressatenspezifisch variierte und das als wichtigster Beitrag zum Framing auf der Meso-Ebene in Abschnitt 5.3 genauer betrachtet wird. Es enthält den zweiten Punkt, in dem die sonst konfrontationsscheue CDU deutlich vor Rot-Grün warnte: die Vergemeinschaftung der europaweiten Staatsschulden durch Eurobonds.

Framing im Politikfeld ,soziale Gerechtigkeit' galt als besondere Herausforderung. Hier hatte die SPD vor Beginn des Wahlkampfs in der öffentlichen Wahrnehmung einen deutlichen Vorsprung vor der CDU. Mit der Priorisierung der beiden anderen Politikfelder war keineswegs entschieden, wie man in der CDU mit dem Thema umgehen sollte, zumal man wusste, dass es das Hauptthema des SPD-Wahlkampfs (sowie von Die Linke und Die Grünen) sein würde. Soll man hier die Konfrontation suchen? Soll man das Thema ignorieren? Oder soll man nach dem Motto „Die CDU ist auch für soziale Gerechtigkeit, und zwar mehr noch als die anderen“"versuchen, die Konkurrenz propagandistisch zu überholen?

Die CDU entschied sich für eine andere Strategie: eine Kombination aus Programmpolitik und Framing. In Parteitagsbeschlüssen und Ankündigungen der Bundeskanzlerin kam es zu sozialpolitischen, arbeitsrechtlichen und wohnungspolitischen Initiativen (Mütterrente, Mindestlohn/Lohnuntergrenze, Mietpreisbremse), die die CDU auf diesem Felde konkurrenzfähig mit der SPD machen sollten. Die erwartbaren emotionalen Reaktionen CDU-relevanter Wähler/innen darauf wurde von den Akteuren als nicht einheitlich eingeschätzt - von Erleichterung bis zu Unmut. 


\begin{tabular}{|c|c|c|c|c|}
\hline Thema & Frame & Attribut & Wert & $\begin{array}{c}\text { Meta-Attribut } \\
\text { EMOTION }\end{array}$ \\
\hline \multirow{9}{*}{$\begin{array}{l}\text { Politik- } \\
\text { felder }\end{array}$} & \multirow{3}{*}{$\begin{array}{l}\text { Frame } 1 \\
\text { Katego- } \\
\text { rie: } \\
\text { Wirt- } \\
\text { schaft } \\
\text { und } \\
\text { Arbeits- } \\
\text { markt }\end{array}$} & $\begin{array}{l}\text { LAGE } \\
\end{array}$ & $\begin{array}{l}\text { geringste Arbeitslosigkeit } \\
\text { seit Jahren, } \\
\text { Exportrekorde }\end{array}$ & $\begin{array}{l}\text { Zufriedenheit, } \\
\text { Zuversicht }\end{array}$ \\
\hline & & PROGRAMM & $\begin{array}{l}\text { weiter so; keine Steuer- } \\
\text { erhöhung! }\end{array}$ & Vertrauen \\
\hline & & $\begin{array}{l}\text { VERANT- } \\
\text { WORTLICH- } \\
\text { KEIT }\end{array}$ & Merkel/CDU/CSU & Vertrauen \\
\hline & \multirow[t]{3}{*}{$\begin{array}{l}\text { Frame } 2 \\
\\
\text { Katego- } \\
\text { rie: } \\
\text { Eurokrise }\end{array}$} & $\begin{array}{l}\text { LAGE } \\
\qquad\end{array}$ & $\begin{array}{l}\text { I. Gefahr durch Staatsver- } \\
\text { schuldung in Euro- } \\
\text { Krisenländern } \\
\text { II. Gegensteuern durch Hilfe } \\
\text { für Krisenstaaten bei Haus- } \\
\text { haltsdisziplin und Struktur-- } \\
\text { reformen }\end{array}$ & $\begin{array}{l}\text { Achtung, Zuver- } \\
\text { sicht }\end{array}$ \\
\hline & & PROGRAMM & $\begin{array}{l}\text { Hilfe für Krisenstaaten nur } \\
\text { bei Haushaltsdisziplin und } \\
\text { Strukturreformen; } \\
\text { keine Eurobonds! }\end{array}$ & $\begin{array}{l}\text { Zuversicht, } \\
\text { Skepsis }\end{array}$ \\
\hline & & $\begin{array}{l}\text { VERANT- } \\
\text { WORTLICH- } \\
\text { KEIT }\end{array}$ & Merkel/CDU/CSU & Vertrauen \\
\hline & \multirow{3}{*}{$\begin{array}{l}\text { Frame } 3 \\
\text { Katego- } \\
\text { rie: } \\
\text { Soziale } \\
\text { Gerech- } \\
\text { tigkeit }\end{array}$} & $\begin{array}{l}\text { LAGE } \\
\end{array}$ & $\begin{array}{l}\text { einige Defizite, z. B. für } \\
\text { Mütter und im Niedrig- } \\
\text { lohnsektor }\end{array}$ & $\begin{array}{l}\ddot{r} \text { rger, Mitleid } \\
\text { Gleichgültigkeit }\end{array}$ \\
\hline & & PROGRAMM & $\begin{array}{l}\text { Mütterrente, Mindestlohn } \\
\text { (Lohnuntergrenze), } \\
\text { Mietpreisbremse }\end{array}$ & $\begin{array}{l}\text { Erleichterung, } \\
\text { Freude, Skepsis, } \\
\text { Unbehagen, } \\
\text { Gleichgültigkeit }\end{array}$ \\
\hline & & $\begin{array}{l}\text { VERANT- } \\
\text { WORTLICH- } \\
\text { KEIT }\end{array}$ & Merkel/CDU/CSU & $\begin{array}{l}\text { Vertrauen, Un- } \\
\text { mut }\end{array}$ \\
\hline
\end{tabular}

Übersicht 8: Ziel-Framecluster ,Politikfelder

Legende:

Pfeil: Kausalrelation (Ursache/Grund $\longrightarrow$ Wirkung/Folge); Ziffer: Relevanz-Rang 
Diese Initiativen wurden nicht unter dem Hochwertwort soziale Gerechtigkeit subsumiert - im Gegenteil. Die Leitlinie war: Wettbewerb in der Sache ja, aber nicht um ein Schlagwort, das als Fahnenwort der politischen Konkurrenz auch bei Gebrauch durch die CDU immer wieder die SPD assoziieren würde. Die defacto-Marginalisierung des Ausdrucks soziale Gerechtigkeit und seiner Bestandteile mit Hilfe semantischer Kniffe war der interessanteste Fall von Framing auf der Mikro-Ebene. In Abschnitt 4.4 wird dies genauer betrachtet.

Die gesamte CDU-Kampagne vermeidet Wahlkampfgetöse und Feldgeschrei. Durchgängig herrscht ein Ton, der in der Selbstdarstellung und ebenso im Umgang mit dem politischen Gegner auf die Erregungen moderater Emotionen setzt - Emotionalisierung auf leisen Sohlen sozusagen.

\subsection{Framing auf der Meso-Ebene: Merkels Begriffsnetz zur Eurokrisenpolitik}

Das Framing zum Thema Eurokrise lag primär bei Angela Merkel. Seit das Thema für sie virulent wurde, hatte sie in fünf Regierungserklärungen zwischen dem 19.5.2010 und dem 14.6.2012 betont: Scheitert der Euro, scheitert Europa (dazu Klein 2014, 123-126). Der Satz bildete das Zentrum der Begründung ihrer Eurokrisenpolitik. Die Substanz der Argumente ist in Begriffen kondensiert, deren Einordnung in das oben erläuterte topische Muster sich problemlos frameanalytisch modellieren lässt (Übersicht 9). Die Konstellation der Topoi ist für die Legitimierung politischen Handelns kanonisch und bildet im Frame eine strukturelle Invariante der Attribute (siehe Abschnitt 1).

Merkel diente der Frame unabhängig von Wahlkämpfen zum Erklären ihrer Politik. Anders als bei kampagnenstrategisch geplanten Zügen ist unsicher, inwieweit die Argumentation - über die Absicht zu überzeugen hinaus - auf Emotionen zielte. Daher ist im Frame ,Eurokrisenpolitik‘ auf das Meta-Attribut EMOTION verzichtet, ebenso auf Tabellenform.

Die in Übersicht 9 präsentierte, zwischen 2010 und 2012 entwickelte Argumentationsstruktur erfuhr mit der Wahlkampagne 2013 in einem entscheidenden Punkt ein ,Reframing': Der Satz Scheitert der Euro, scheitert Europa verschwand aus der Begründung Merkel'scher Krisenpolitik. Die Möglichkeit eines Scheiterns auch nur zu erwähnen, passte nicht in das ,positive Denken' der feel-good-Kampagne. Statt negative Konsequenzen zu beschwören, las und hörte man nun im 
topischen Begründungscluster zur Eurokrisenpolitik ein neues Positiv-Datum: Reformen erfolgreich auf dem Weg. Mitzudenken war: ,vor allem dank der deutschen Kanzlerin“.

DATEN: Eurokrise als Staatsschuldenkrise: drohende Zahlungsunfähigkeit von EuroStaaten; verschärfter globaler Wettbewerb; Deutschland als Stabilitätsanker und Wachstumsmotor

URSACHEN: übermäßige Staatsverschuldung; mangelnde Wettbewerbsfähigkeit KONSEQUENZ: Scheitert der Euro, scheitert Europa.

PRINZIPIEN: Solidarität bei Eigenverantwortung, Hilfe nur bei eigener Leistung ZIELE: Stabilitätsunion, Euro als starke und stabile Währung, Wettbewerbsfähigkeit, mehr Wachstum und Beschäftigung, (abgelehnt: Transferunion / Schuldenunion)

POLITISCHES HANDELN: Strukturreformen, Haushaltssanierung, Schuldenbremse, Fiskalpakt, Bankenunion, ESM, Finanztransaktionssteuer (abgelehnt: Eurobonds)

Übersicht 9: Frame Eurokrisenpolitik

Legende: In Kapitälchen: Attribute; Kursiv in Normalschreibung: wörtlich zitierte Begriffe, Begriffskombinationen sowie der Satz Scheitert der Euro, scheitert Europa; Klammer: stigmatisierende Begriffe für abgelehnte, primär der SPD zugeschriebene politische Konzepte; strichlinierte Linie: Schlussfolgerungssymbol (Übergang von den Argument- zu den Konklusionsbegriffen)

Das Merkel'sche Begriffsnetz war im Rahmen von Debatten und Verhandlungen im professionellen Bereich politischer Institutionen entstanden. Interessierten Bürgerinnen und Bürgern war es durchaus vertraut, weil die politischen Medien es transportierten und weitgehend übernommen hatten. In der Wahlkampagne 2013 splittete die Kanzlerin beim Thema Europa Begrifflichkeit und Sprachstil adressatenspezifisch: In TV- und Presse-Interviews, wo sie mit politisch überdurchschnittlich interessiertem Publikum rechnen konnte, blieb sie (mit der skizzierten inhaltlichen Verschiebung) bei dem seit 2010 entwickelten Begriffsinventar. In ihrer Standardwahlrede für ein weniger sachkundiges Publikum verzichtete sie auf die Spezifizierung der komplizierten Probleme der Eurorettung. In der oben zitierten Passage (vgl. Abschnitt 4.2.2.2) rechtfertigt sie ihr Bemühen um Europa grundlegender und verständlicher. Sie kontrastiert zwei Mini-Frames: das Europa der Streitereien der Regierungschefs mit dem Europa unstrittiger Werte 
(Demokratie, Pressefreiheit, Meinungsfreit...), und betont die weit größere Relevanz dieser Gemeinsamkeit, gestützt durch den Hinweis auf das, was in Ägypten in Syrien und in anderen Ländern los ist.

\subsection{Framing auf der Mikro-Ebene: Marginalisierung eines Fahnenworts der SPD ${ }^{24}$}

$\mathrm{Zu}$ Beginn des Wahlkampfs machte es die CDU nervös, als die SPD soziale Gerechtigkeit thematisch in den Mittelpunkt rückte. Wie sollte sie auf ein Thema reagieren, das der Gegner mit populären Vorschlägen konkretisierte: gesetzlicher Mindestlohn, Mietpreisbremse etc.? Meinungsumfragen signalisierten breite Zustimmung und machten deutlich: Soziale Gerechtigkeit wird vor allem der SPD zugeordnet - trotz Agenda 2010.

Sprachstrategen wie Biedenkopf oder Geißler, die legendären CDU-Generalsekretäre unter Kohl, hätten wohl empfohlen, den ,Begriff für die CDU zu ,besetzen'. Es hätte bedeutet, ihn häufig in den Mund zu nehmen und mit bisherigen Leistungen der Regierung in Verbindung zu bringen. Die CDU reagiert 2013 umgekehrt. Sie ergreift eigene programmatische Initiativen (Mütterrente, tariflicher Mindestlohn, Mietpreisbremse). Aber sie hütet sich vor dem rhetorischen Schlagabtausch um die Herrschaft über das Hochwertwort soziale Gerechtigkeit. So konnte man dem sozialpolitischen Singularitätsanspruch der SPD begegnen und darüber hinaus die Bedeutung des sozialdemokratischen Symbolworts mindern. Die Kampagnenakteure hatten Ergebnisse der Kognitiven Linguistik und der Sprachpsychologie zur Kenntnis genommen (z. B. Lakoff 2004 und Kahneman 2012): Je öfter man den Ausdruck soziale Gerechtigkeit in den Mund nähme, desto mehr käme die SPD konnotativ ins Spiel.

Andererseits kann keine Partei den Begriff ganz aus ihrem Wortschatz tilgen. Dazu ist er für Deutschland als Sozialstaat zu zentral. Wie lässt man einen Begriff gleichzeitig verschwinden und nicht verschwinden? Dazu entwickelten Kampagnenakteure eine lexikalische Strategie durch Kombination dreier Verfahren:

- Minimalübernahme (= einmalige, aber relevante Verwendung im Grundsatzteil des Wahlprogramms): So verbindet die Soziale Marktwirtschaft in einzigartiger Weise die Vorteile einer Marktwirtschaft mit der Verpflichtung

${ }^{24}$ Vgl. Klein (2013, 200 f.) 
zur sozialen Gerechtigkeit. ${ }^{25}$ Der Satz klingt im Rahmen eines Bekenntnisses zur Sozialen Marktwirtschaft als Leitbild der CDU (und der CSU) ideologisch gewichtig. Man schützt sich so vor dem Vorwurf, soziale Gerechtigkeit gering zu schätzen. Aber in der Masse der Sätze und Texte geht er unter, zumal der Begriff in der Kampagne nirgendwo sonst auftaucht.

- Umgehung durch Konkretisierung: Statt soziale Gerechtigkeit als Oberbegriff den einschlägigen Einzelmaßnahmen wie Mütterrente, tariflicher Mindestlohn, Mietpreisbremse etc. in Überschriften oder anderweitig überzuordnen, wird einfach auf einen Oberbegriff verzichtet. Hier wird deutlich, dass es den Akteuren nicht darum ging, das Konzept, das mit dem Ausdruck soziale Gerechtigkeit verbunden ist und dem die genannten Initiativen der CDU durchaus entsprachen, grundsätzlich in Frage zu stellen, sondern einen weithin positiv besetzten Ausdruck in den eigenen Texten und Reden zu meiden, weil er mit der politischen Konkurrenz assoziiert wird.

- Diffundierende Bezeichnungskonkurrenz: Es bleibt nicht beim Vermeiden des gegnerische Fahnenwortes. Die Vermeidungsstrategie trifft auch die Bestandteile sozial und gerecht. Sie werden zwar nicht völlig aus dem Sprachgebrauch der CDU verbannt, aber überwiegend durch bedeutungsähnliche Wörter ersetzt: Statt sozial heißt es meist anständig, sicher, verantwortungsvoll, ordentlich $\mathrm{u}$. $̈$. und statt gerecht überwiegend fair, anständig. ${ }^{26}$ Es ist der Versuch, Markenwörter aus dem Ideologievokabular der SPD zu ertränken in bedeutungsähnlichen Allerweltswörtern mit emotional positiver Anmutung.

\section{Frameanalytische Zusammenführung und eine Schlussbemerkung}

Wie in Abschnitt 3 angedeutet, lassen sich die in den bisherigen Abschnitten getrennt voneinander präsentierten Frames und Subframes (Übersichten 2-9) zu einer Frame-Subframe-Struktur ,CDU-Bundestagswahlkampagne 2013` zusam-

\footnotetext{
25 CDU/CSU (2013): Gemeinsam erfolgreich für Deutschland. Regierungsprogramm 2013-2017, 17.

${ }^{26}$ Die entsprechende Empfehlung des „Leitfadens für gute Sprache im Wahlkampf“ der CDU (Leitfaden, 13) wurde im Wahlprogramm und anderen Texten so praktiziert.
} 
menfügen (Übersicht 10). Darin wird der rekursive Charakter von Frames deutlich. Die Frames der Makro- und Mikroebene sind als Sub-Subframes in die Struktur eingeklinkt, ohne dass die Wege der Rekursion über etwaige Zwischenstufen im Einzelnen expliziert werden.

\begin{tabular}{|c|c|c|}
\hline \multicolumn{3}{|c|}{ Kategorie: CDU-Bundestagswahlkampagne 2013} \\
\hline Attribut & $\begin{array}{l}\text { Werte spezifizierende Sub-Frames } \\
\text { und Sub-Subframes }\end{array}$ & $\begin{array}{l}\text { Meta-Attribut } \\
\text { EMOTION }^{27}\end{array}$ \\
\hline WÄHLERSCHAFT & $\begin{array}{l}\text { CDU-relevante Wahlberechtigte (Ü 2) } \\
\longrightarrow \text { politikrelevante Tugenden (Ü 3) }\end{array}$ & $\begin{array}{l}\text { überwiegend } \\
\text { moderat }\end{array}$ \\
\hline \multirow{3}{*}{ POLITIKFELD } & Wirtschaft/Arbeitsmarkt (Ü4, Ü 8) & \multirow{3}{*}{ moderat } \\
\hline & $\begin{array}{l}\text { Eurokrise (Ü 4, Ü 8) } \\
\begin{array}{l}\longrightarrow \\
\text { Eurokrisenpolitik (Ü 9, Erläute- } \\
\text { rungen zu Ü9) [Meso-Ebene] }\end{array}\end{array}$ & \\
\hline & $\begin{array}{l}\text { Soziale Gerechtigkeit (Ü 4, Ü 8) } \\
\qquad \begin{array}{l}\longrightarrow \\
\text { SPD-Fahnenwort-Marginalisie- } \\
\text { rung (Abschn. 4.4) [Mikro-Ebene] }\end{array}\end{array}$ & \\
\hline \multirow[b]{2}{*}{ KANDIDAT } & Merkel (Ü 5, Ü 7) & \multirow{2}{*}{ moderat } \\
\hline & Steinbrück (Ü 6) & \\
\hline
\end{tabular}

Übersicht 10: Frame-Subframe-Struktur,CDU-Bundestagswahlkampagne 2013“ Legende: Übersicht: Ü; Spezifizierung durch Sub-Subframe:

Unter kommunikationswissenschaftlichen Aspekten handelt es sich bei dem Beitrag um eine Kombination aus Produktions- und Produktforschung. Framing-Forschung, vor allem die medien-orientierte, ist überwiegend Wirkungsforschung. Aus dieser Tradition heraus liegt die Frage nahe, ob das strategische Framing verfangen und die angestrebten Wirkungen erzielt hat? Diese Frage mit traditionellen Mitteln der empirischen Sozialforschung zu untersuchen, würde bei einem so

${ }^{27}$ Die Emotions-Werte sind für den Ist-Zustand unterstellt und als Ziel-Zustand angestrebt. 
komplexen Gegenstand wie einer Wahlkampagne erhebliche Mittel und etliche Forschungsprojekte erfordern. In unserem Falle liegt allerdings ein gewichtiges Indiz anderer Art zumindest für generelle Wirk-Tendenzen vor: das Wahlergebnis. Zwar pflegen Wahlsiege viele Mütter und Väter zu haben, doch wäre es bei einem so klaren und hohen Wahlsieg wie dem der Union 2013 wenig rational anzunehmen, dass alles mögliche Andere für den Erfolg der Kampagne verantwortlich war, nur nicht das strategische Framing.

\section{Literatur}

Barsalou, Lawrence W. (1992): Frames, concepts, and conceptual fields. In: Kittay, Eva / Lehrer, Adrienne (eds.): Frames, fields, and contrasts. New essays in semantic and lexical organization. Hillsdale, NJ: Lawrence Erlbaum, 21-74.

Barsalou, Lawrence W. (1993). Flexibility, structure, and linguistic vagary in concepts: Manifestations of a compositional system of perceptual symbols. In: Collins, Alan. F. / Gathercole, Susan E. / Conway, Martin A. / Morris, Peter (eds.): Theories of memory. London: Lawrence Erlbaum, 29-101.

Berger, Peter L. / Luckmann, Thomas (1966): The Social Construction of Reality. Garden City NY: Doubleday.

Busse, Dietrich (2008): Semantische Rahmenanalyse als Methode der Juristischen Semantik. Das verstehensrelevante Wissen als Gegenstand semantischer Analyse. In: Christensen, Ralph / Pieroth, Bodo (Hrsg.): Rechtstheorie in rechtspraktischer Absicht. Freundesgabe zum 70. Geburtstag von Friedrich Müller. Berlin: Duncker \& Humblot, 35-55.

Busse, Dietrich (2012): Frame-Semantik. Ein Kompendium. Berlin / Boston: de Gruyter.

Entman, Robert M. (1991): Framing US-Coverage of International News: Contrasts in Narratives of the KAL and Iran Air Incidents. In: Journal of Communication 41, H. 4, 6-27.

Entman, Robert M. (1993): Framing: Toward Clarification of a Fractured Paradigm. In: Journal of Communication 43, H. 4, 51-58.

Fillmore, Charles J. (1977): Scenes-and-Frames Semantics. In: Zampolli, Antonio (ed.): Linguistic Structures Processing. Amsterdam / New York / Oxford (North Holland): Elsevier Science, 55-81. 
Fillmore, Charles J. / Atkins, Beryl T. (1992): Towards a frame-based lexicon: the case of RISK. In: Kittay, Eva / Lehrer, Adrienne (eds.): Frames, Fields, and Contrasts. New Essays in Semantic and Lexical Organization. Hillsdale NJ: Lawrence Erlbaum Publishers, 75-102.

Fraas, Claudia (1996): Gebrauchswandel und Bedeutungsvarianz in Textnetzen. Die Konzepte ,Identität‘ und ,deutsche Einheit“ im Diskurs zur deutschen Einheit. Tübingen: Gunter Narr.

Hermanns, Fritz (1986): Appellfunktion und Wörterbuch. Ein lexikographischer Versuch. In: Wiegand, Herbert Ernst (Hrsg.): Studien zur neuhochdeutschen Lexikographie VI.1 (= Germanistische Linguistik 84-86). Hildesheim / Zürich / New York: Olms, 151-182.

Holly, Werner (2001): ,Frame‘ als Werkzeug historisch-semantischer Textanalyse. Eine Debattenrede des Chemnitzer Paulskirchen-Abgeordneten Eisenstuck. In: Diekmannshenke, Hajo / Meißner, Iris (Hrsg.): Politische Kommunikation im historischen Wandel. Tübingen: Stauffenburg, 125-146.

Kahneman, Daniel (2012): Schnelles Denken, langsames Denken. München: Siedler.

Klein, Josef (1991): Kann man „Begriffe besetzen’? Zur linguistischen Differenzierung einer plakativen politischen Metapher. In: Liedtke, Frank / Wengeler, Martin / Böke, Karin (Hrsg.): Begriffe besetzen. Strategien des Sprachgebrauchs in der Politik. Opladen: Westdeutscher Verlag, 44-69.

Klein, Josef (1995): Asyl-Diskurs. Konflikte und Blockaden in Politik, Medien und Alltagswelt. In: Reiher, Ruth (Hrsg.): Sprache im Konflikt. Berlin / New York: de Gruyter, 15-71.

Klein, Josef (1999): „Frame“ als semantischer Theoriebegriff und als wissensdiagnostisches Instrumentarium. In: Pohl, Inge (Hrsg.): Interdisziplinarität und Methodenpluralismus in der Semantikforschung. Frankfurt a.M. / Berlin: Peter Lang, 157-183.

Klein, Josef (2000): Komplexe topische Muster: Vom Einzeltopos zur diskurstypspezifischen Topos-Konfiguration. In: Schirren, Thomas / Ueding, Gert (Hrsg.): Topik und Rhetorik. Tübingen: Niemeyer, 623-649. 
Klein, Josef (2002): Topik und Frametheorie als argumentations- und begriffsgeschichtliche Instrumente, dargestellt am Kolonialdiskurs. In: Cherubim, Dieter./ Jakob, Karlheinz / Linke, Angelika (Hrsg.): Neue deutsche Sprachgeschichte: Mentalitätsgeschichtliche, kultur- und sozialgeschichtliche $\mathrm{Zu}-$ gänge. Berlin / New York: de Gruyter, 167-181.

Klein, Josef (2003): Politische Rede. In: Ueding, Gert (Hrsg.): Historisches Wörterbuch der Rhetorik. Bd. 6, Tübingen: Niemeyer, 1465-1521.

Klein, Josef (2007): Texte mit globaler Resonanz. Auch ein Beitrag zu diskursivem ,Kampf der Kulturen'. In: aptum. Zs. für Sprachkritik und Sprachkultur. Heft 2, 122-138.

Klein, Josef (2013): (Fast) alles ist gut - mit Angela Merkel als Kanzlerin. Wie die CDU die Wahlkampfsprache unspektakulär und erfolgreich revolutioniert. In: aptum. Zeitschrift für Sprachkritik und Sprachkultur. 9, H.03, 195-207.

Klein, Josef (2014): Grundlagen der Politolinguistik. Berlin: Frank \& Timme.

Klein, Josef / Meißner, Iris (1999): Wirtschaft im Kopf. Begriffskompetenz und Einstellungen junger Erwachsener bei Wirtschaftsthemen im Medienkontext. Frankfurt a. M. / Berlin [u. a.]: Peter Lang.

Konerding, Klaus Peter (1993): Frames und lexikalisches Bedeutungswissen. Tübingen: Niemeyer.

Kuck, Kristin / Römer, David (2012): Metaphern und Argumentationsmuster im Mediendiskurs zur ,Finanzkrise‘. In: Peltzer, Anja / Lämmle, Kathrin / Wagenknecht, Andreas (Hrsg.): Krise, Cash und Kommunikation. Die Finanzkrise in den Medien. Konstanz / München: UVK, 71-93.

Kühne, Rinaldo / Schemer, Christian (2014): Emotionale Framing-Effekte auf politische Einstellungen und Partizipationsbereitschaft: In: Marcinkowski, Frank (Hrsg.): Framing als politischer Prozess. Beiträge zum Deutungskampf in der politischen Kommunikation. Baden-Baden: Nomos, 195-210.

Kuhlmann, Christoph (1999): Die öffentliche Begründung politischen Handelns. Zur Argumentationsrationalität in der politischen Massenkommunikation. Opladen / Wiesbaden: Westdeutscher Verlag.

Lakoff, George (2004): Don't think of an elefant. Know your values and frame the debate. White River Junktion (Vermont): Chelsea Green Publishing.

Lakoff, George / Wehling, Elisabeth (2007): Auf leisen Sohlen ins Gehirn. Politische Sprache und ihre heimliche Macht. Heidelberg: Carl-Auer-Verlag. 
Luntz, Frank (2006): The New American Lexicon. (seit 2006 als Raubdruck kursierendes ,playbook' zum Couching republikanischer Politiker).

Luntz, Frank (2009): What Americans Really Want . . Really. New York: Hyperion.

Matthes, Jörg (2014): Framing. Baden-Baden: Nomos.

Minsky, Marvin (1974): A Framework for Representing Knowledge. (= MIT-AI Laboratory Memo 306, June 1974). Wiederabdruck in: Winston, P.H. (ed.): The Psychology of Computer Vision. New York: McGraw-Hill, 211-278.

Römer, David / Wengeler, Martin (2013): „Wirtschaftskrisen“ begründen / mit „Wirtschaftskrisen“ legitimieren. Ein diskurshistorischer Vergleich. In: Wengeler, Martin / Ziem, Alexander (Hrsg.): Sprachliche Konstruktionen von Krisen. Bremen: Hempen Verlag, 269-288.

Schwarz-Friesel, Monika (2007): Sprache und Emotionen. Tübingen / Basel: A. Francke.

Searle, John R. (1997): The Construction of Social Reality. New York: The Free Press.

Ziem, Alexander (2008): Frames und sprachliches Wissen. Kognitive Aspekte der semantischen Kompetenz. Berlin / New York: de Gruyter. 